\title{
Forest and Plantation Soil Microbiomes Differ in Their Capacity to Suppress Feedback Between Geosmithia morbida and Rhizosphere Pathogens of Juglans nigra Seedlings
}

\author{
Geoffrey M. Williams ${ }^{1}$ and Matthew D. Ginzel ${ }^{1,2, \dagger}$ \\ ${ }^{1}$ Department of Forestry and Natural Resources, Purdue University, West Lafayette, IN 47907 \\ ${ }^{2}$ Department of Entomology, Purdue University, West Lafayette, IN 47907
}

Accepted for publication 28 July 2021.

\section{ABSTRACT}

Thousand cankers disease (TCD) is hypothesized to have a greater impact on eastern black walnut (Juglans nigra) in urban forests and plantations compared with natural forest stands. Along with other factors such as resource availability, the phytobiome could partly account for observed differences in disease severity across management regimes. We investigated the extent to which J. nigra-associated soil microbiomes from plantations and natural forests modulate (i) the amount of necrosis caused by Geosmithia morbida in 1-year-old seedlings, and (ii) relative abundance of rhizosphere endophytes and opportunistic pathogens Fusarium and Rhizoctonia in response to aboveground inoculation with $G$. morbida. Our results suggest that the microbiome from natural forest soil in central Indiana suppresses Fusarium spp. and is indirectly suppressive of G. morbida. Natural forest soil had a greater ability to reduce the size of necrotic area caused by G. morbida compared with steam-treated soil. Inoculating stems of seedlings with $G$. morbida induced a shift in fungal community composition in the rhizosphere, including Fusarium and Rhizoctonia spp.; however, the direction and magnitude of the shift depended on whether seedlings were amended with forest, plantation, or steamtreated soil. In a companion experiment, necrotic area in G. morbida-inoculated seedlings was twice as high in seedlings grown from seeds that were treated with Fusarium solani relative to those grown from seed treated with water. Our findings support the hypothesis that TCD severity can be modulated by host-mediated feedback between above- and belowground pathogens, as well as by microbial interactions in the rhizosphere.

Keywords: black walnut, disease-suppressive soil, endophyte, Fusarium, latent pathogen, microbiome, phyllosphere, plant pathology, plant-soil feedback, rhizosphere, thousand cankers disease, Trichoderma
The etiology of tree diseases can be best understood within a framework of predisposing conditions, proximate or primary agents,

${ }^{\dagger}$ Corresponding author: M. Ginzel; mginzel@purdue.edu

Author contributions: G.M.W. and M.D.G. designed the study and wrote the manuscript together. Laboratory, field, and greenhouse work and data analysis were completed by G.M.W.

Current address for G. M. Williams: U.S. Forest Service, International Programs, Washington, D. C. 20005

Funding: This work was supported by the Department of Forestry and Natural Resources, Purdue University through a Fred M. van Eck Scholarship to G. Williams and an Investments in Excellence Small Grant awarded to G. M. Williams and M. D. Ginzel. Publication of this article was funded in part by Purdue University Libraries Open Access Publishing Fund. This research was in partial fulfillment of a doctoral degree for G. M. Williams from Purdue University.

The author(s) declare no conflict of interest.

(c) 2022 The American Phytopathological Society and ultimate causes of mortality that includes both biotic and abiotic factors (Manion 1981; Tainter and Baker 1996). This framework is particularly relevant when considering diseases where severity is highly variable across environmental conditions, such as thousand cankers disease (TCD) of eastern black walnut (Juglans nigra L.). The proximate cause of TCD, an emergent disease of J. nigra and other Juglans and Pterocarya spp., is attack by the walnut twig beetle Pityophthorus juglandis Blackman and necrosis caused by its primary fungal symbiont, Geosmithia morbida M. Kolař́k, Freeland, C. Utley \& Tisserat (Ploetz et al. 2013; Tisserat et al. 2009).

Additional factors, including site quality or the activity of other microorganisms, can predispose hosts to disease or contribute to later stages of decline and ultimate mortality. TCD appears to be most severe in urban forests and managed plantations, whereas natural forest populations of J. nigra in its native range have remained relatively unaffected, even in areas where the pathogen and vector have been detected (Juzwik et al. 2020; Seybold et al. 2019). Urban forests are frequently subject to invasive pest outbreaks due to 
predisposing factors such as connectivity with transportation pathways, higher ambient and soil temperatures, increased wind and drought exposure, and physical damage (Klein and Perkins 1987; Tello et al. 2005). Natural forest landscapes are likely to be more resilient to disease compared with managed plantations due to higher biological and structural diversity (Coyle et al. 2005; Ennos 2015; Wingfield et al. 2015; Jactel et al. 2021).

Environmental differences among managed plantations, urban forests, and natural forests can also include dissimilarities in the composition and function of the bulk soil microbiome and host phytobiome. Such differences in the microbiome may include the relative abundance of other pathogens or beneficial endophytes that could play roles in the etiology of disease (Arnold et al. 2000; Carroll 1988; Porras-Alfaro and Bayman 2011). Pathogens other than G. morbida are frequently found in soil around TCDsymptomatic walnut trees or in tissues surrounding subdermal cankers (Gazis et al. 2018; Kasson et al. 2014; Lauritzen 2018; Onufrak et al. 2020). In fact, a role for Fusarium solani species complex (FSSC) (Summerell 2019) (Sordariomycetes: Hypocreales: Nectriaceae) in the etiology of later stages of TCD was recognized in the first description of the disease (Tisserat et al. 2009). FSSC spp. were frequently isolated by Tisserat et al. (2009) from the boles of trees in the later stages of decline, indicating that these generalist pathogens might contribute to the ultimate causes of mortality of trees with TCD. Culture-based studies of TCD-symptomatic trees have since uncovered a diversity of $F$. solani, $F$. fujikuroi, $F$. lateritium, and $F$. tricinctum species complexes (Summerell 2019) associated with cankers (Gazis et al. 2018; Kasson et al. 2014; Lauritzen 2018). In particular, FSSC sp. 25 is found in close association with G. morbida in TCD-symptomatic walnut trees in the United States and Italy but does not increase the size of cankers when coinoculated with G. morbida, nor does it cause larger cankers alone compared with water controls (Juzwik et al. 2020; Kasson et al. 2014; Montecchio et al. 2015; Sitz et al. 2017). Thus, the impact of these and other canker-causing pathogens on the etiology of TCD remains unclear. Nevertheless, other studies suggest that multitrophic interactions between the host and primary and secondary pathogens lead to the development of plant disease and are regulated by the microbiome (Lamichhane and Venturi 2015; van Gils et al. 2017).

Trees in urban and plantation forests exist in a state of heightened physiological stress due to abiotic factors, management activities, and contrasting levels of herbivory and disease (Coyle et al. 2005; Durrant and Dong 2004; Van Loon 1997). This stress may also condition the host microbiome and susceptibility to disease (Yuan et al. 2018). In particular, limited genetic and species diversity in plantations and orchards might lead to the accumulation of pests and secondary pathogens (Coyle et al. 2005; Ennos 2015; Yang et al. 2012). These same secondary pathogens, including Fusarium, related Nectriaceae, and Rhizoctonia spp. (Agaricomycetes: Cantharellales: Ceratobasidiaceae) are thought to be major contributors to plant-soil feedbacks, in which plants condition soil biota to the benefit (positive feedback) or detriment (negative feedback) of conspecifics (Bennett and Klironomos 2019; Klironomos 2002; McKenry 1999). These negative feedbacks are also responsible for replant diseases of orchard crops in the western United States where TCD is severe (Browne et al. 2006; Mazzola 1998; Schmidt et al. 2010). Plant-soil feedbacks and belowground pests and pathogens can also promote aboveground herbivores, including bark beetles (Aukema et al. 2010; Hertert et al. 1975; Kaplan et al. 2018; van Gils et al. 2017). Therefore, it is possible that feedbacks between G. morbida and soil-dwelling microbiota such as FSSC spp. play a role in TCD etiology as predisposing factors or ultimate causes of mortality.

In natural forests where TCD has been slower to establish, $J$. nigra might benefit from favorable associations that contribute to host resilience such as mycorrhizae or other mutualistic fungi and bacteria (Gazis et al. 2018; Jung et al. 2012; Pineda et al. 2017). For example, Trichoderma spp. have been employed as a biological control for plant diseases, and were found in higher abundance and diversity in $P$. juglandis galleries in the eastern compared with the western United States (Gazis et al. 2018). Accumulation of pathogens may also lead to recruitment of beneficial, antagonistic microbiota (Schlatter et al. 2017). In addition to build-up of pathogens such as Gibberella zeae (Schwein.) Petch (syn. F. graminearum Schwabe) in soils around TCD-infected trees, beneficial fungi such as mycoparasites were found in soils around noninfected trees (Onufrak et al. 2020). The extent to which beneficial microbes and feedback between aboveground pathogens such as Geosmithia morbida and soil biota influence host susceptibility to and severity of bark beetle-fungal diseases such as TCD has not been explored. Basic knowledge of these processes could be translated to management strategies to improve interactions of trees with pests and pathogens in their environment (Busby et al. 2017).

We conducted two experiments to test the hypothesis that the soil microbiome modifies susceptibility or resistance of $J$. nigra seedlings to G. morbida under greenhouse conditions. In our first experiment, untreated potting mix was amended with live or steamtreated soil to (i) determine the extent to which forest and plantation soil microbiomes affect the size and healing of cankers caused by G. morbida and (ii) assess the effect of live and steam-treated soil amendments on feedback between aboveground G. morbida infection and the microbiome of seedling roots. We were particularly interested in the response of Fusarium and Rhizoctonia spp., soilborne pathogens associated with negative soil feedback (Cesarano et al. 2017), to inoculation with G. morbida. In a second experiment, we treated seed with two endophytic fungi isolated from roots in the first experiment to determine the extent to which they affected canker growth after seedlings were inoculated with G. morbida. One isolate (FSSC sp.) was a putative disease synergist and the other (Trichoderma sp.) was a putative biological control fungus. We hypothesized that FSSC sp. would increase the size and decrease healing of cankers and that Trichoderma sp. would limit root colonization by FSSC sp. or canker growth.

\section{MATERIALS AND METHODS}

Live soil amendment experiment. Soil collection. Soils were collected on 21 May 2018 from four plots (each 3 by $3 \mathrm{~m}$ ): two located in two different plantations of J. nigra of ages 50 years $\left(40^{\circ} 25^{\prime} 51.6^{\prime \prime} \mathrm{N}, 87^{\circ} 02^{\prime} 25.2^{\prime \prime} \mathrm{W}\right)$ and 28 years $\left(40^{\circ} 25^{\prime} 60.0^{\prime \prime} \mathrm{N}\right.$, $87^{\circ} 02^{\prime} 07.3^{\prime \prime} \mathrm{W}$ ) at the Martell experimental forest (West Lafayette, IN) and two plots that were each located below two different $100+$ year old $J$. nigra trees growing in mixed hardwood stands adjacent to each of the two plantations. Approximately 500 liters (bulk volume) of soil was removed from the top approximately $8 \mathrm{~cm}$ of the plots, sifted through $1.5-\mathrm{cm}$ wire mesh to remove debris, and mixed by type (forest and plantation) to obtain a composite sample of each type. A subsample of approximately 120 liters (bulk volume) of each soil type was kept at $4^{\circ} \mathrm{C}$ for 3 to 5 days prior to planting.

Potting of bare-root seedlings with live soil amendments. On 22 May 2018, we planted 120 1-year-old J. nigra seedlings grown at the Vallonia nursery (Indiana Department of Natural Resources) from mixed, open-pollinated seed collected in Indiana. The nursery beds at the Vallonia nursery are fumigated with methyl bromide (336 kg/ha) and chloropicrin (56 kg/ha) (James McKenna, personal communication). Prior to planting, root systems were trimmed to stimulate root growth and remove damaged roots and washed with water and antimicrobial dish soap to remove nursery soil. We planted seedlings in Metro-Mix 560 Sun-Coir (560SC) tree potting 
media (Sun Gro Horticulture, Agawam, MA, U.S.A.) in new 9.6-liter TP818 Treepots (Stuewe and Sons, Corvallis, OR, U.S.A.). We chose not to sterilize the 560SC potting mix because it would result in the loss of nutrients and soil structure, and can lead to misleading inferences (Trevors 1996; van Gils et al. 2017). To ensure that a sufficient amount of live soil inoculum and substrate were present to transfer potential disease suppressiveness (Schlatter et al. 2017), potting mix was amended $20 \%$ by bulk volume with live soil from forest $(n=40)$ or plantation $(n=40)$ soil. As a control soil amendment, we used a 50-50 mixture of forest and plantation soil that had been heat pasteurized in a 54 Soil Steamer (Hummert International, Topeka, KS, U.S.A.) twice for $5 \mathrm{~h}$ with steam at 30 PSIG and $274^{\circ} \mathrm{C}$ with an intervening 24-h cooling period. The steam-treated soil mixture was also added to pots at a $20 \%$ bulk volume ratio like the other treatments $(n=40)$. Seedlings were grown in the Entomology Environmental Laboratory greenhouses at Purdue University, West Lafayette, IN, U.S.A. Greenhouse temperatures ranged from 20 to $40^{\circ} \mathrm{C}$. No supplemental fertilization was provided beyond what was available in the amended 560SC tree potting media and soil amendments. All plants were watered to saturation as needed over the course of the growing period.

Inoculation of seedlings with $\mathrm{G}$. morbida and measurement of necrosis and healing. In the greenhouse, seedlings were inoculated with G. morbida 43 days after transplanting (4 July) following the methods of Utley et al. (2013). Cultures of isolate IN-66 from Brown County, IN (Juzwik et al. 2015) were grown for 1 week on one-eighth strength (1/8-strength) potato dextrose agar (PDA) ( $4.9 \mathrm{~g}$ of PDA powder $+13.1 \mathrm{~g}$ of agar per 1 liter of $\mathrm{H}_{2} \mathrm{O}$ ) (Thermo Fisher Scientific, Waltham, MA, U.S.A.). A 6-mm-diameter corkborer was used to remove the outer bark and phloem $20 \mathrm{~cm}$ above the root collar. In the resulting wound, seedlings from each soil amendment treatment received a 6-mm-diameter plug of either $G$. morbida ( $n=30$ per soil amendment treatment) or sterile $1 / 8$ strength PDA (control inoculation, $n=10$ ). Across the three soil treatments, in total, 90 seedlings were inoculated with G. morbida and 30 were inoculated with sterile $1 / 8$-strength PDA alone as a control. After inoculation, wounds were sealed with parafilm and duct tape.

Seedlings were harvested between 18 and 30 September 2018 (76 to 88 days postinoculation). Bark and phloem was carefully removed from the outer sapwood, and inner phloem was imaged with a flatbed scanner to allow precise quantification of necrotic area by manually tracing canker margins using ImageJ 1.51 (United States National Institutes of Health). To collect data on host response, canker healing was scored on an ordinal scale (Fig. 1A).

Isolation of fungi from roots. Roots were harvested from 10 G. morbida-inoculated and 10 agar-only control plants within each soil amendment treatment. A total sample of approximately 80 root sections (approximately $2 \mathrm{~cm}$ each) were harvested at random from 10 primary or secondary lateral roots of each plant. Our root surface sterilization procedure was modified from Arnold et al. (2000) and consisted of three successive 30-s washes in sterile distilled water (SDW) with a vortex, followed by rinsing with agitation in $70 \% \mathrm{EtOH}$ (15 s), $2 \% \mathrm{NaOCl}(2 \mathrm{~min})$, and $70 \% \mathrm{EtOH}$ (15 s); a final rinse in SDW; and air drying on sterile paper towel. Ten surface-sterilized root segments per plant were plated onto each of the following five types of media: (i) half-strength (1/2-strength) PDA++ (Tisserat et al. 2009), (ii) $2.5 \mathrm{ppm}$ malachite green agar (Castellá et al. 1997), (iii) glucose yeast extract rose bengal agar (Newhouse and Hunter 1983), (iv) "modified PDA" formulated as described by Vargas Gil et al. (2009), and (v) natamycin-ampicillin-rifampicin-pentochloronitrobenzene cornmeal agar at $5 \mathrm{mg} / \mathrm{liter}$ (Jeffers and Martin 1986). In total, a subset of 1,000 root pieces ( $n=50$ per plant) were plated onto five different selective growth media ( $n=10$ per media per plant). The latter four selective media were chosen to promote the growth of known seedling pathogens or beneficial fungi: Fusarium, Cylindrocladium, Trichoderma, and Phytophthora-Pythium spp., respectively (Mielke and Ostry 2004). To verify the effectiveness of the surface-sterilization procedure, imprint plates were made on quarter-strength (1/4-strength) PDA from root samples collected from eight seedlings from each soil treatment for a total of 24 imprint plates (Ridout et al. 2017). All
A

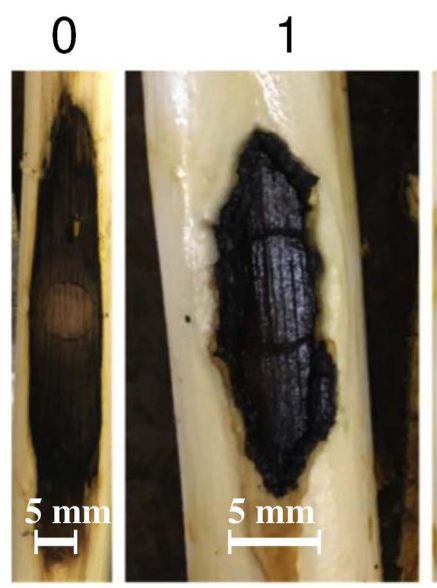

2

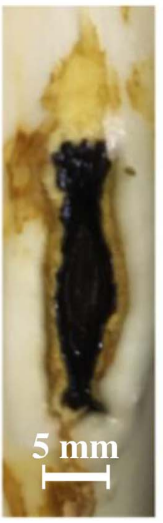

3

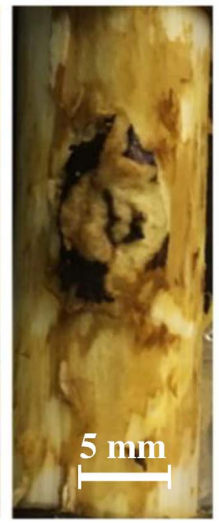

B

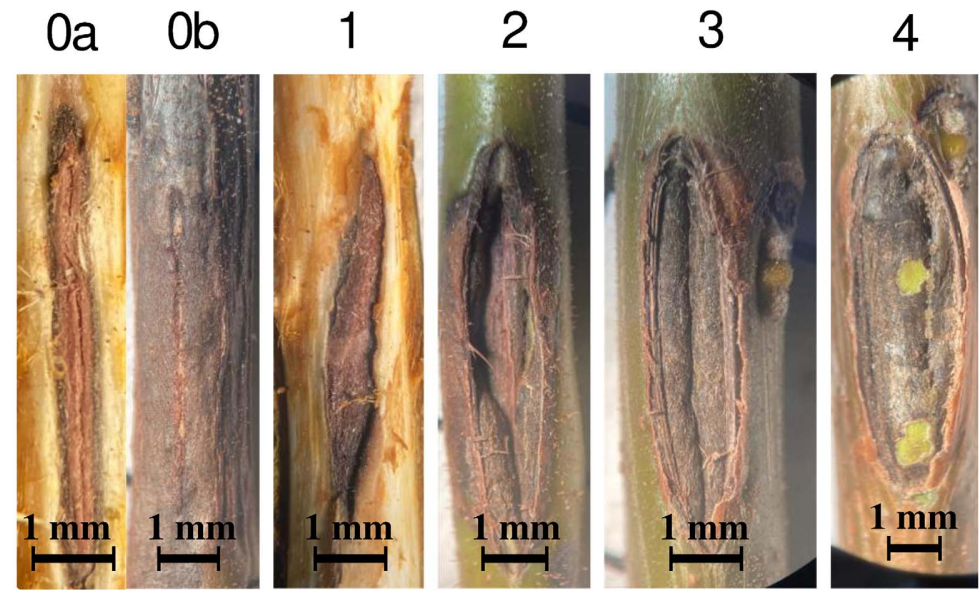

Fig. 1. Key to ratings of inoculation wound healing. The amount of reactive host tissue response and healing of wounds increases from left to right in each panel. Different scoring systems were used to score healing of wounds after inoculation with Geosmithia morbida in the live soil amendment experiment, which employed 6-mm plugs to inoculate 1-year old seedlings (A) and the endophyte inoculation experiment, which employed spore suspensions to inoculate 3-month old seedlings (B). A, Canker healing scale for plug inoculations in live soil amendment experiment (sapwood shown): $0=$ no raised reactive sapwood border around canker margin; 1 = raised boundary around canker margin without covering original wound; $2=$ wound partly covered by reactive tissue; and $3=$ wound is completely healed. $\mathbf{B}$, Canker healing scale for spore suspension inoculations in endophyte inoculation experiment (outer bark shown unless otherwise indicated): $0=$ no raised reactive sapwood border around canker margin, (a) sapwood and (b) outer bark shown; 1 = raised boundary around canker margin without covering original wound (sapwood shown); 2 = wound partly covered by reactive tissue; $3=$ sides of wound closed but not joined by new tissue; and $4=$ sides of closed wound joined by formation of healing scar. 
plates were checked semiweekly for 2 months and all fungal colonies from plated roots were subcultured on 1/4-strength PDA until pure cultures were obtained to be used for extraction of DNA.

$P C R$ and bioinformatics. To obtain molecular-based taxonomic assignments of fungal isolates from root subcultures, DNA was extracted from subcultures following Lee and Taylor (1990) and subjected to PCR amplification of the internal transcribed spacer (ITS) region. Each PCR (25 $\mu \mathrm{l}$ each) contained $\mathrm{MgCl}_{2}(1.5 \mathrm{mM})$, forward and reverse primers ITS5 and ITS4 (White et al. 1990), dNTPs $(0.2 \mu \mathrm{M}$ each), PlatinumTaq DNA Polymerase $(0.1 \mu \mathrm{l})$, DNA template $(1 \mu \mathrm{l})$, and PlatinumTaq buffer $(1 \times)$. A touchdown thermocycle profile was employed with denaturation at $94^{\circ} \mathrm{C}$ for $4 \mathrm{~min} ; 5$ cycles of $94^{\circ} \mathrm{C}$ for $30 \mathrm{~s}, 48^{\circ} \mathrm{C}$ for $30 \mathrm{~s}$ and $72^{\circ} \mathrm{C}$ for $1 \mathrm{~min} ; 5$ cycles of $94^{\circ} \mathrm{C}$ for $30 \mathrm{~s}, 47^{\circ} \mathrm{C}$ for $30 \mathrm{~s}$, and $72^{\circ} \mathrm{C}$ for $30 \mathrm{~s}$; 20 cycles of $94^{\circ} \mathrm{C}$ for $30 \mathrm{~s}, 46.3^{\circ} \mathrm{C}$ for $30 \mathrm{~s}$ and $72^{\circ} \mathrm{C}$ for $30 \mathrm{~s}$; and final extension at $72^{\circ} \mathrm{C}$ for $10 \mathrm{~min}$. PCR products were cleaned with a PureLink Pro 96 PCR Purification Kit (Invitrogen, Waltham, MA, U.S.A.) and sequenced with standard protocols at the Purdue Genomics Facility (West Lafayette, IN, U.S.A.) on an 3730XL DNA Analyzer (Applied Biosystems, Waltham, MA, U.S.A.).

Assembly of forward and reverse sequences, quality control, and trimming were performed with the Chromaseq package and base tools in Mesquite (Edgar 2004; Maddison and Maddison 2019a, b). Trimmed assembled sequences were pairwise aligned, furthestneighbor clustered at $95 \%$ similarity, and assigned to taxonomy concepts from the UNITE database version 02.02.2019 in mothur 1.39.3 (Nilsson et al. 2019; Schloss et al. 2009) (https://unite.ut.ee). Sequences of interest were also aligned via basic local alignment (BLAST) to the NCBI (Altschul 1990) (https://blast.ncbi.nlm.nih. gov/Blast.cgi). Sequences have been deposited to GenBank under accessions MW301139 and MW326227 to MW326432.

Statistical analyses. All statistical analyses were performed in R 3.6.1 (R Core Team 2019). Pathogenicity of G. morbida isolate IN-66 relative to agar-only control inoculation was confirmed separately within soil treatment groups with one-way analyses of variance (ANOVA) on Box-Cox transformed necrotic area (Venables and Ripley 2002). Subsequent analyses of canker necrotic area and healing across soil treatments were performed for $G$. morbida-inoculated plants only.

The effect of soil amendment on canker growth was analyzed with ANOVA after a Box-Cox transformation and removing outliers with Cook's distance $>4 / \mathrm{n}$ (Fox and Weisberg 2019). Among the 30 control seedlings that were inoculated with PDA alone $(n=10$ per soil treatment), 1 seedling in the sterile soil treatment died. Among the G. morbida-inoculated seedlings ( $n=30$ per soil amendment treatment), 10 seedlings were left out of the analysis: 2 outliers from the plantation soil, 2 from the forest soil amendment groups, and 6 seedlings that died, leaving a total of $n=80 \mathrm{G}$. morbida-inoculated seedlings. This removal resulted in a slightly unbalanced number of $G$. morbida-inoculated seedlings between the steam-treated $(n=27)$, plantation $(n=26)$, and forest $(n=27)$ soil amendment groups for analysis of necrotic area among soil treatments. Marginal and conditional $R^{2}$ were calculated (Nakagawa et al. 2017). Pairwise group comparisons of necrotic area among treatments were performed with a Tukey posthoc test. The effect of soil amendment on canker healing rating was tested with proportional-odds logistic regression (i.e., ordinal regression) and drop-in-deviance Wald $\chi^{2}$ tests (ANODEV). Healing was compared among groups with unadjusted regression contrasts (Venables and Ripley 2002).

Effects of soil amendment, stem inoculation treatments, and their interaction on community composition were tested with 9,999-permutational multivariate analysis of variance on Jaccard distances (ADONIS) (Oksanen et al. 2018). For the ADONIS, rare operational taxonomic units (OTUs) represented by five or fewer isolates were removed from the dataset along with samples rendered empty by the removal of the rare OTUs, resulting in a slightly unbalanced design. Because ADONIS uses marginal sum of squares, the analysis was repeated for both possible orders of main effects to ensure robust inferences.

For individual taxa, isolation and reisolation colony counts of fungi from soil and plant tissues were analyzed with quasipoisson log-link generalized linear models and ANODEV with overdispersion-adjusted contrasts. Colony counts of isolates assigned to the genera Fusarium (O'Donnell et al. 2020) and Rhizoctonia were considered as a function of G. morbida inoculation, soil treatment, and their interaction. $R^{2}$ for all ordinal and quasipoisson regressions were calculated with the drop-in-deviance method (McFadden 1974).

Endophyte inoculation experiment. Inoculation of seedlings with root endophytes and G. morbida. On 12 April 2019, stratified half-sibling seed from Hardwood Tree Improvement and Regeneration Center J. nigra accession number $288(n=31)$ were sown in steam-treated potting mix. Once seed germinated, they were inoculated with isolates of a Fusarium sp. (Rh-217) and a Trichoderma sp. (Rh-366) from the first experiment. These isolates were chosen because they were identified as potentially pathogenic or pathogenantagonistic endophytic fungal species, respectively. Seed were washed thoroughly in water followed by $2 \% \mathrm{NaOCl}(2 \mathrm{~min})$. Seed were germinated in 12-cm pots containing potting media (560SC) with Osmocote fertilizer (Scotts Co., Marysville, OH, U.S.A.) at $3.125 \mathrm{~g} /$ liter of bulk soil and lime at $11.25 \mathrm{~g} /$ liter of bulk soil that had been autoclaved three times for $2 \mathrm{~h}$ with two intervening days of cool-down between cycles. Seed were inoculated with fungi at approximately $60 \%$ germination, 25 days after sowing (7 May) with isolates of F. solani (Mart.) Sacc. (Rh-217) and Trichoderma sp. (Rh-366) from the previous experiment grown in $2 \%$ malt extract broth at $30^{\circ} \mathrm{C}$ for 25 days. Cultures were homogenized in a blender and $75 \mathrm{ml}$ was added to each pot. To control for the potential effects of waste, mycotoxins, or residual nutrients from liquid cultures of each fungus on the soil microbiomes or plant physiology, we added $75 \mathrm{ml}$ of filtrate obtained by passing homogenate through a $22-\mu \mathrm{m}$ filter (Nalgene Nunc, Rochester, NY, U.S.A.) to each pot from the other fungus treatment(s) (Table 1). Absence of viable fungal propagules was verified by spreading $10 \mu \mathrm{l}$ of filtrate on five 1/4-strength PDA plates.

Seedlings were moved to 6.2-liter TP616 Treepots (Stuewe and Sons) 29 days after soil inoculations and inoculated with G. morbida 34 days after transplanting ( 8 July), at an age of approximately 3 months. Seedlings were inoculated with spore suspensions because seedling stem diameters were too small for the use of agar plugs. The spore suspension was prepared from 60-day-old cultures of IN-66 grown on 1/8-strength PDA and adjusted to $100,000 \mu \mathrm{l}^{-1}$ in a 1:10,000 volume solution of Tween 20 and SDW. The spore suspension $(5 \mu \mathrm{l})$ was pipetted into three rectilinear wounds $(5 \mathrm{~mm})$ made by a utility knife in the phloem, until the wounds were filled and overflowing, at 5,10 , and $15 \mathrm{~cm}$ above the root collar on the north side of the stem, and sealed with parafilm.

Seedlings were harvested 80 to 87 days after inoculation (26 September to 3 October). To measure necrosis and healing at the conclusion of the experiment, outer bark was shaved away with a utility knife to expose maximum phloem necrosis, which was imaged on a flatbed scanner and measured with ImageJ as described above. Canker healing was scored on an ordinal scale (Fig. 1B).

Reisolation of Fusarium and Trichoderma spp. From each plant, $402-\mathrm{cm}$ root segments were collected, surface sterilized as described above, and placed on selective Fusarium agar (SFA) $(n=20$ pieces per plant; Leslie and Summerell 2008) and Trichoderma selective media ( $n=20$ pieces per plant) (Vargas Gil et al. 2009). Cultures 
from roots were checked semiweekly for 30 days and all fungal colonies that grew from root pieces were classified to morphospecies and counted. From identified morphospecies, two colonies were subcultured as vouchers within each morphospecies, plant, and media type. For quantification of FSSC from bulk soil, soil was collected from each pot with a sterile 50-ml Falcon tube and a subsample of $3.75 \mathrm{~g}$ was serially diluted to a final concentration of $15 \mathrm{~g} /$ liter in a 1:100,000 volume solution of Tween 20 and SDW. We plated $800-\mu 1$ aliquots of the resulting soil dilution on four plates of peptone-pentonitrochlorobenzene agar (Leslie and Summerell 2008) per plant. After 7 days, colonies that grew from soil dilutions were counted and classified to morphospecies within sets of plates from five plants at a time, and two colonies were subcultured as vouchers for each morphospecies for molecularbased taxonomic assignments. To reisolate and quantify FSSC and G. morbida from cankers, 120 approximately $10-\mathrm{mm}^{2}$ sections of necrotic phloem ( $n=4$ to 6 per plant) were washed briefly in $70 \%$ EtOH (10 s) followed by SDW (10 s), and placed on 1/2-strength PDA++. Fungi were maintained on 1/4-strength PDA or SFA and examined under a light microscope at $\times 400$ (roots and soil) or as a slide mount at $\times 1,000$ (necrotic lesions) magnification, and classified as F. solani, other Fusarium spp., Trichoderma sp., G. morbida, or "other" prior to DNA extraction.

PCR and bioinformatics. DNA was extracted from cultures as described above. To identify Fusarium spp. from the endophyte inoculation experiment, the translation elongation factor 1- $\alpha$ (TEF1a) gene was also amplified with primers EF-1a-EF1 and EF-1aEF2 (O'Donnell et al. 1998) in addition to amplification of the ITS region as described above. TEF1 reactions consisted of $30 \mathrm{ml}$ with the same concentrations of reagents to ITS reactions described above but with modification to $\mathrm{MgCl}(2.5 \mathrm{mM})$, forward and reverse primer $(0.25 \mu \mathrm{M})$, and template $(2 \mu \mathrm{l})$. For TEF, thermocycle profile included denaturation at $94^{\circ} \mathrm{C}$ for $1 \mathrm{~min}$; 35 cycles of $94^{\circ} \mathrm{C}$ for $30 \mathrm{~s}, 59^{\circ} \mathrm{C}$ for $45 \mathrm{~s}$, and $72^{\circ} \mathrm{C}$ for $1 \mathrm{~min}$; and final extension at $72^{\circ} \mathrm{C}$ for $10 \mathrm{~min}$. Products from the endophyte inoculation experiment were purified and sequenced at GeneWiz corporation (South Plainfield, NJ, U.S.A.).

Sequences were processed and aligned as described above and deposited to GenBank under accessions MW300958 to MW301040 and MW456941 to MW457023. Sequences of interest were also aligned via basic local alignment (BLAST) to the Fusarium-ID database (Geiser et al. 2004) (http://isolate.fusariumdb.org/blast. php). To identify putative Fusarium isolates recovered in the endophyte inoculation experiment and determine their relationship to the isolate $\mathrm{Rh}-217$ used in inoculations and other species, we built a phylogenetic tree that included vouchered accessions from the Fusarium multilocus sequence typing database (O'Donnell et al. 2012) (https://fusarium.mycobank.org). A majority-rule consensus tree was made in PAUP from 1,000 bootstrap trees generated from concatenated ITS-TEF-1a alignments in RAxML under a GTR+CAT model, and visualized in R 3.6.1 (R Core Team 2019; Stamatakis 2014; Swofford 2003).

Statistical analyses. Generalized linear mixed models (GLMM) of canker necrosis and healing were fit with random effects for plant nested within treatment. Apart from the inclusion of random effects, the GLMM were fit as described above for the ANOVA on necrosis and proportional odds logistic regression on healing. Marginal and conditional $R^{2}$ were calculated for fixed and fixed plus random effects (Nakagawa et al. 2017). To test for the effect of endophyte treatment on reisolation rate of Fusarium morphotypes with ITS+TEF1 voucher sequences identical to Rh-217, separate quasipoisson and ANODEV analyses were performed as described above at the level of each substrate from which isolates were obtained (i.e., cankers, roots, and soil).

\section{RESULTS}

Live soil amendment experiment. Effect of soil amendment on necrosis and healing. In our live soil amendment experiment, amending potting mix with forest-associated soil biota led to smaller cankers caused by G. morbida in seedlings; however, the effects of plantation soil on necrotic area were intermediate and nonsignificant (Fig. 2A). Soil amendment had a significant effect on area of necrosis caused by $G$. morbida $\left(P=0.011 ; d f_{1,2}=2,77\right.$; $\left.R^{2}=0.11\right)$. Amending seedlings with forest soil reduced the size of cankers by $17.2 \pm 5.6 \%\left(5.8 \pm 1.9 \mathrm{~mm}^{2}[ \pm\right.$ denotes 1 standard error]; Tukey $P=0.008)$ relative to the steam-treated soil amendment treatment (Fig. 2A). Necrotic area for seedlings that received the plantation soil amendment was intermediate and not significantly different from those that were amended with steam-treated soil (Tukey $P=0.202$ ) or forest soil (Tukey $P=0.385$ ). G. morbida inoculation led to an increase in necrotic area compared with agar-only control inoculations among seedlings that were amended with forest $\left(10.4 \pm 3.7 \mathrm{~mm}^{2} ; P=0.007 ; d f=37\right)$, plantation $\left(13.6 \pm 3.2 \mathrm{~mm}^{2} ; P<0.001 ; d f=36\right)$, and steam-treated $(18.3 \pm$ $\left.3.3 \mathrm{~mm}^{2} ; P<0.001 ; d f=34\right)$ soil.

Soil amendment also had a significant effect on healing of inoculation wounds $\left(P=0.024 ; d f=2 ; R^{2}=0.04\right)$ and seedlings amended with forest soil had the highest probability of healing (Fig. 2B). On average, seedlings that were amended with forest and

TABLE 1

Design of endophyte inoculation experiment

\begin{tabular}{|c|c|c|c|}
\hline Treatment & Homogenate & Filtrate & Replicates $^{a}$ \\
\hline FSSC $^{b}$ & FSSC Rh-217 & Trichoderma Rh-366 & 5 \\
\hline Trichoderma $^{\mathrm{c}}$ & Trichoderma Rh-366 & FSSC Rh-217 & 5 \\
\hline Control & None & FSSC Rh-217 + Trichoderma Rh-366 & 4 \\
\hline Nonsterile $^{d}$ & None & None & 3 \\
\hline
\end{tabular}

60 Phytobiomes Journal 
plantation soil had 4.5-fold (95\% confidence interval [CI] [1.3, 14.6]; $P=0.014)$ and 3.7 -fold $(95 \%$ CI $[1.1,12.3] ; P=0.032)$ higher odds, respectively, of being in a higher healing rating category relative to those that received steam-treated soil (Fig. 2B).

Effects of soil amendment and G. morbida inoculation on rhizosphere fungal community. We recovered full-length ITS sequences for 203 fungal isolates from roots of J. nigra seedlings across all treatments and media types: forest $(n=66)$; plantation $(n=60)$; and steam-treated $(n=77)$ soil amendments (GenBank accessions MW326227 to MW326432). Bacteria grew on 9 of 24 (37.5\%) imprint plates but only one imprint plate $(4 \%)$ had fungal growth. Isolates from roots of seedlings grown with forest, plantation, and steam-treated soil amendments clustered into 18, 20, and 18 OTUs, respectively. In total, 39 OTUs were classified to at least 20 genera and 24 families across 15 known orders of Fungi (Fig. 3A). Only 9 OTUs occurred in all three treatments.

The influence of aboveground inoculation on belowground community structure depended on soil amendment treatment (Fig. 3A). The composition of the fungal rhizosphere microbiome of seedlings that received plantation or steam-treated soil amendments shifted in response to inoculation with $G$. morbida. By contrast, the fungal rhizosphere microbiome of seedlings grown with forest soil was relatively stable regardless of G. morbida inoculation (Fig. 3A). Overall, isolates assigned to the order Hypocreales, including FSSC and Trichoderma, were less abundant in the trees grown in soil amended with forest soil than the other treatments (Fig. 3A and B). Within the plantation soil amendment treatment, however, Hypocreales members were more abundant and Cantherellales less abundant in G. morbida-inoculated seedlings than the control-inoculated seedlings. In contrast, within the steam-treated soil amendment Hypocreales members were less abundant and Cantherellales more abundant in the G. morbida-inoculated seedlings than the controlinoculated seedlings (Fig. 3A). These observations suggest that members of Cantherellales and Hypocreales in our experiment may have antagonized each other and that their abundance depended on both $G$. morbida inoculation and soil amendments.

Regardless of the order of main effect terms in ADONIS, neither soil amendment $\left(d f_{1}=2\right)$ nor $G$. morbida inoculation $\left(d f_{1}=1\right)$ significantly accounted for variation in community composition of culturable root endophytes $\left(P>0.6 ; d f_{2}=38\right.$; Pseudo- $\left.R^{2}<0.05\right)$, nor did they have a significant interaction $\left(P=0.19 ; d f_{1,2}=2,36\right.$;
Pseudo- $\left.R^{2}=0.06\right)$. Removal of rare species $(n<6)$ from the dataset left only eight OTUs for ADONIS, which were assigned by UNITE to FSSC sp. (OTU1), Rhizoctonia sp. (OTU2), Lophiotremataceae sp. (OTU3), Apodus sp. (OTU4), Paraphoma sp. (OTU5), Zopfiella sp. (OTU6), Tulasnellaceae sp. (OTU7), and Trichoderma sp. (OTU8). Isolates assigned to Zopfiella were most abundant in the seedlings amended with forest soil and least abundant in those amended with plantation soil; by contrast, isolates belonging to FSSC spp. were least abundant in those amended with forest soil (Fig. 3B).

Aboveground inoculation of stems with G. morbida influenced the colonization of roots by Fusarium and Rhizoctonia spp. (Fig. 4). In particular, in the presence of plantation-associated soil biota, aboveground $G$. morbida facilitated colonization by belowground Fusarium spp. (Fig. 4A). Whether Fusarium and Rhizoctonia spp. increased or decreased in abundance in the rhizosphere in response to inoculation with G. morbida depended on the soil amendment given to the seedlings, as shown by a significant interaction effect on Fusarium spp. infection $(P=0.049 ; d f=2)$. Though the interaction effect on Rhizoctonia spp. was nonsignificant $(P=0.203 ; d f=2)$, aboveground $G$. morbida inoculation led to positive feedback on the infection of roots by Rhizoctonia spp. in the steam-treated amendment seedlings and negative feedback on Rhizoctonia spp. in the plantation soil-amended seedlings. This observation contrasts with negative feedback on Fusarium spp. in the steam-treated amendment seedlings and positive feedback on Fusarium spp. in the plantation soil-amended seedlings. Moreover, in the rhizospheres of forest soil-amended seedlings, feedback on Fusarium spp. was diminished compared with the other treatments and there was no difference in Rhizoctonia spp. between seedlings inoculated with G. morbida and PDA alone (Fig. 4A and B).

Pairwise contrasts were not significant after accounting for substantial overdispersion. However, in the rhizospheres of seedlings amended with plantation soil, genus Fusarium was isolated $8.5 \pm$ 13.6 times more frequently from roots of $G$. morbida-inoculated seedlings compared with agar-only controls. This difference was less pronounced for seedlings amended with forest soil, where Fusarium was isolated only $4.0 \pm 6.8$ times more frequently from roots of G. morbida-inoculated plants than controls inoculated with agar only (Fig. 4A). In seedlings with steam-treated soil, Fusarium spp. were isolated $4.2 \pm 4.5$ times more frequently from roots of

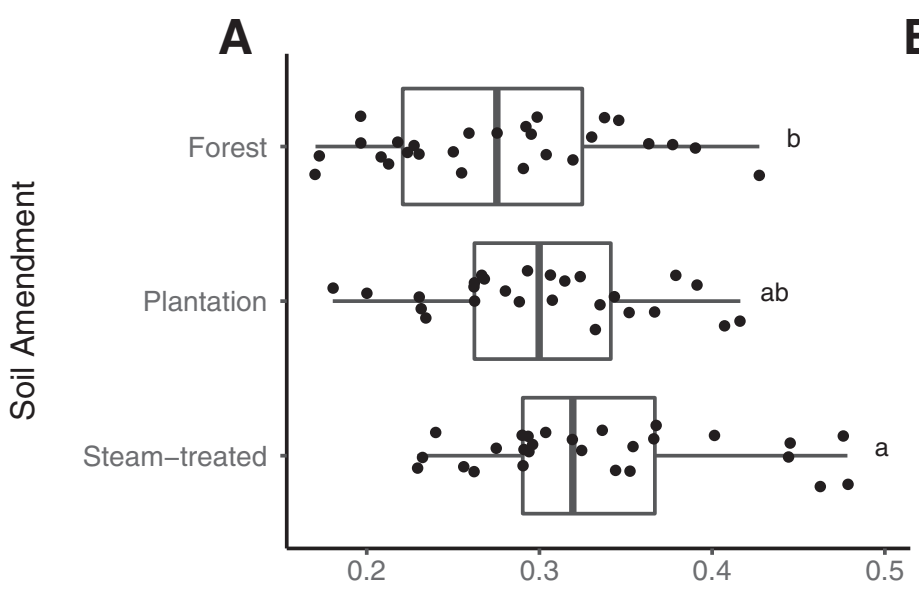

Necrotic Area $\left(\mathrm{cm}^{2}\right)$
B

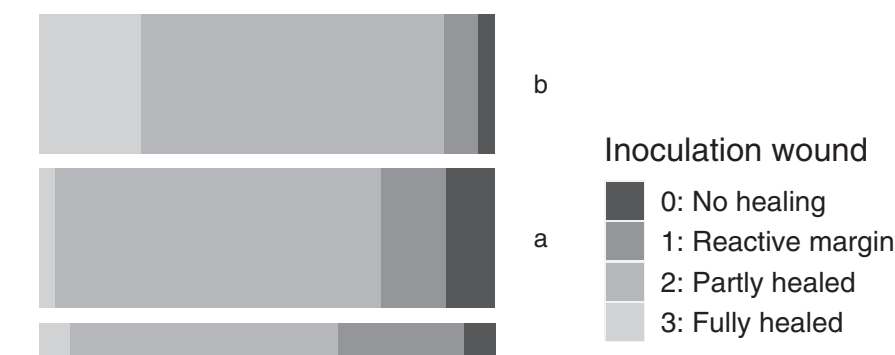

a

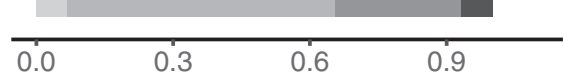

Proportion of replicates

Fig. 2. A, Necrotic area and B, healing of cankers caused by Geosmithia morbida in seedlings that received forest, plantation, and steam-treated soil amendments. Different letters in plot A denote significant Tukey-adjusted contrasts from a Box-Cox $(\lambda=0.9)$ transformed linear model. Outliers not shown. Different letters in plot B denote significant unadjusted contrasts from a log-link proportional odds model. 
agar-only control-inoculated plants compared with those inoculated with G. morbida.

Endophyte inoculation experiment. Effect of FSSC sp. and Trichoderma $s p$. on necrosis and canker healing. FSSC isolate Rh-217 appeared to originate from potting mix and predisposed seedlings to greater damage from the pathogen $G$. morbida. Upon inoculation with G. morbida, prior inoculation with FSSC Rh-217 facilitated increased necrotic area and suppression of canker healing
(Fig. 5A and B). The area of necrosis caused by G. morbida depended on seed treatment $\left(P<0.001 ; d f=4 ; R_{\text {fix }}^{2}=0.43\right.$; $\left.R_{\text {fix }+ \text { rand }}^{2}=0.57\right)$. Necrotic area was greater in stems of plants that had been inoculated with FSSC Rh-217 (Fig. 5A). Necrotic area was $112.5 \pm 35.7 \%$ higher in FSSC-inoculated plants than in sterile control plants that received filtrates only $\left(7.66 \pm 2.43 \mathrm{~mm}^{2}\right.$; Tukey $P=0.011)$. Necrotic canker area was $163.2 \pm 42.2 \%$ higher in the plants grown in nonsterile soil without treatment relative to the
A
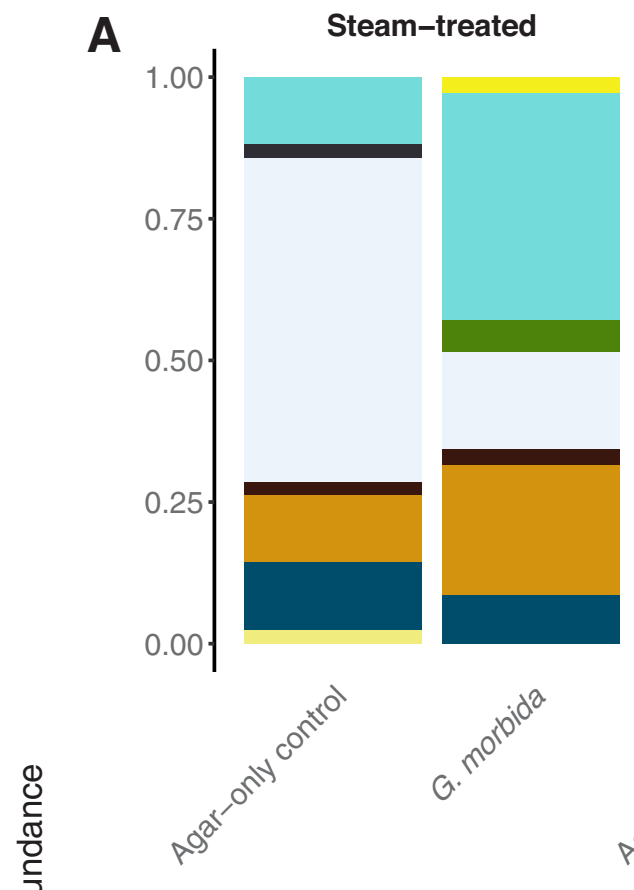

B

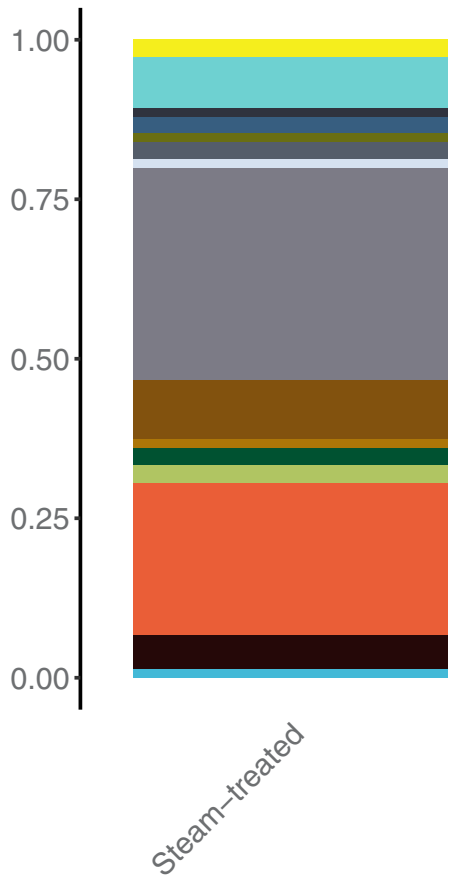

Plantation
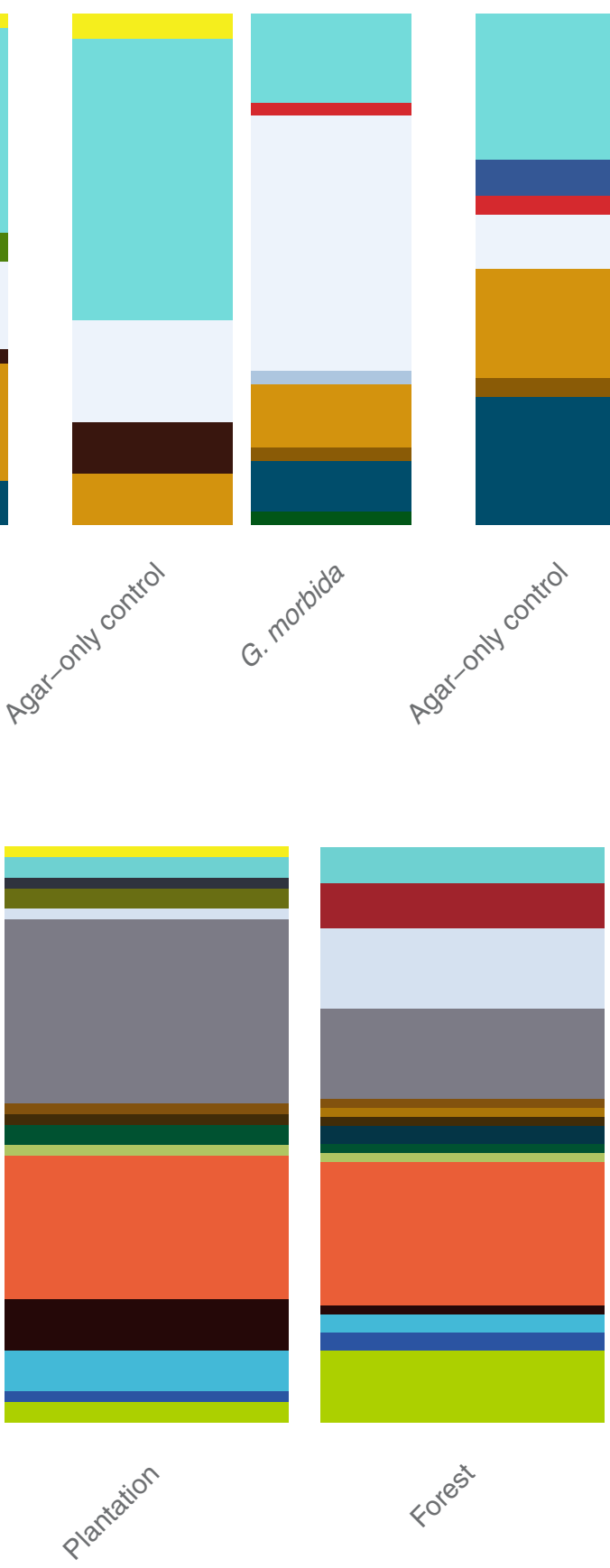

Forest
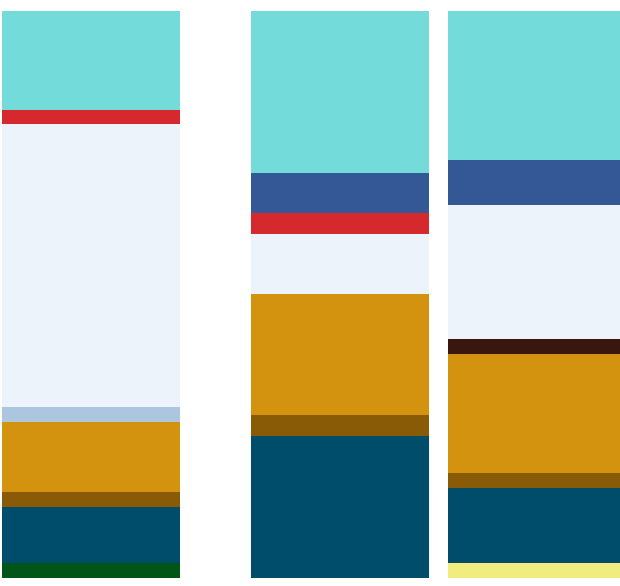

Order
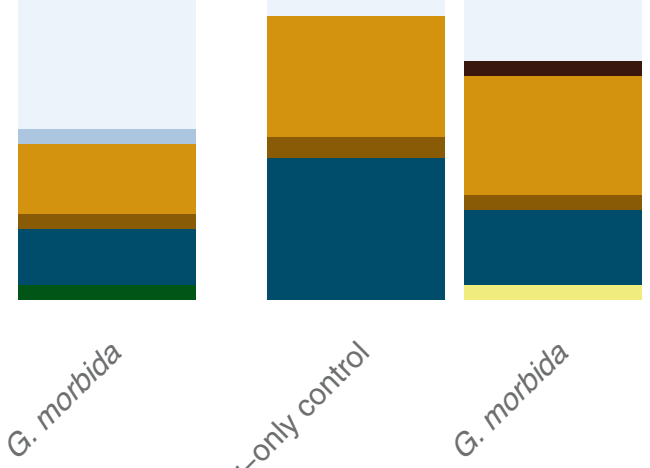

Ascomycota_sp Cantharellales

Capnodiales Diaporthales Fungi_sp Glomerellales Hypocreales Magnaporthales Microascales Pleosporales Savoryellales Sordariales Thelebolales Xylariales
Genus

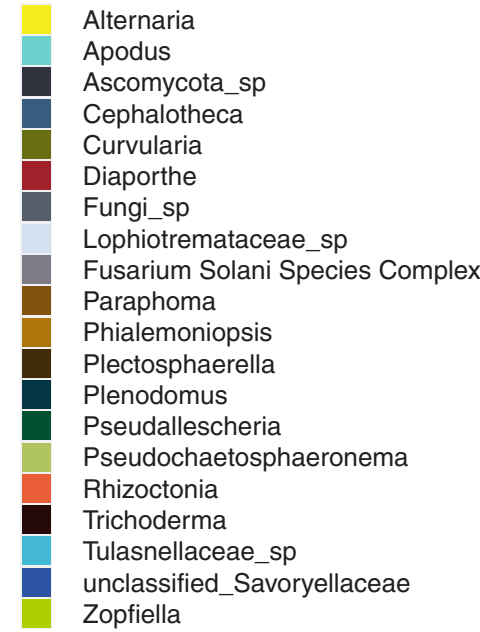

Fig. 3. Composition of microbiome by soil amendment and Geosmithia morbida inoculation treatment. A, Relative abundance of UNITE database fungal order assignments of isolated endophyte operational taxonomic units based on internal transcribed spacer (ITS) sequences from roots of seedlings grown in potting mix amended with steam-treated, plantation, and forest soil and inoculated with G. morbida or agar-only control. B, Relative abundance of UNITE database fungal genus assignments based on ITS sequences of nonsingleton $(n>1)$ endophytes isolated from roots of seedlings grown in potting mix amended with steam-treated, plantation, and forest soil. 
sterile control plants $\left(11.1 \pm 2.9 \mathrm{~mm}^{2}\right.$; Tukey $\left.P<0.001\right)$. Overall canker size did not differ between the plants grown in nonsterile soil without FSSC treatment and those that received the FSSC inoculation treatment (Tukey $P=0.337$ ). Coinoculation of germinating seedlings with both FSSC Rh-217 and Trichoderma Rh-366 together did not result in smaller cankers compared with FSSC Rh-217 alone (Tukey $P=0.997$ ).

Trichoderma increased healing of inoculation wounds, whereas healing was decreased by Fusarium. Endophyte treatment also had a significant effect on canker healing $\left(P=0.013 ; d f=4 ; R_{\text {fix }}^{2}=\right.$ $\left.0.10 ; R_{\text {fix }+ \text { rand }}=0.17\right)$. On average, inoculation wounds of control seedlings had 27.4-fold (95\% CI [1.5, 509.3]; $P=0.026)$ higher odds of being in a more advanced healing category relative to those inoculated with FSSC Rh-217 (Fig. 5B). Control plants had 24.1-fold higher odds of healing relative to plants grown in nonsterile soil and not inoculated (95\% CI [0.7, 796.2]; $P=0.075$ ). Although inoculation with FSSC Rh-217 alone or with Trichoderma Rh-366 resulted in similarly sized cankers, coinoculation with Trichoderma Rh-366 increased the odds of being in a higher healing category 70.6 -fold (95\% CI $[4.9,1016.8]$; $P=0.002)$ compared with seedlings inoculated with FSSC Rh-217 alone. In total, 8 of 21 (38\%) coinoculations with FSSC Rh-217 and Trichoderma Rh-366 led to full healing of the G. morbida inoculation wound, compared with just 1 across all other treatments.

Reisolation of fungi used in inoculations. Isolate Rh-217 was found to be most closely related to FSSC sp. 35-a, 25-a, or 25-b (Fig. 6) and its ITS sequence differed by only one base insertion from FSSC sp. 25 isolated from TCD-symptomatic trees in Italy
A

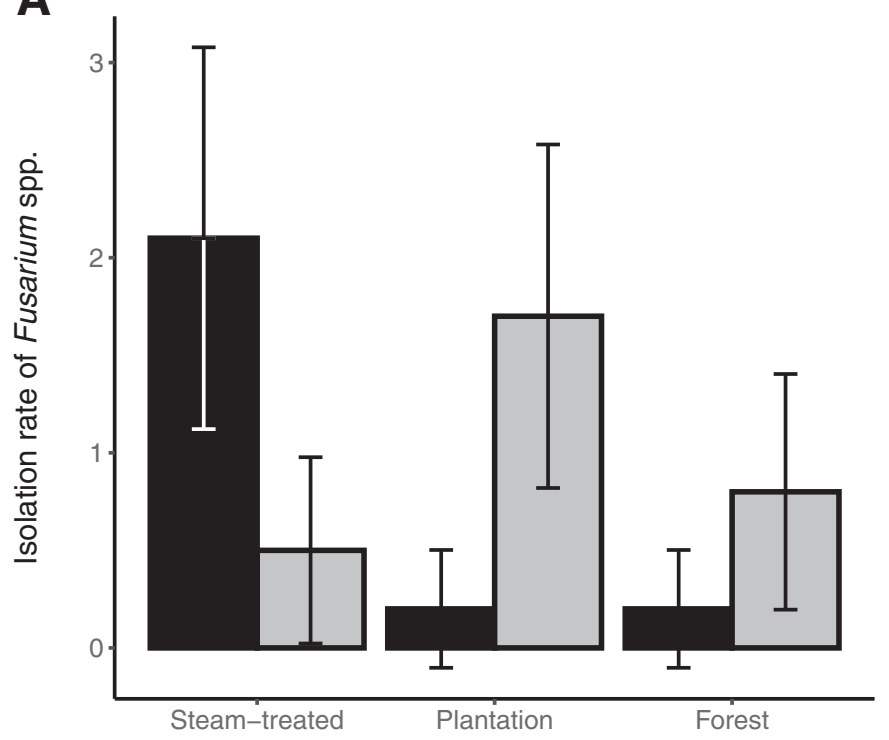

Soil Amendment
B

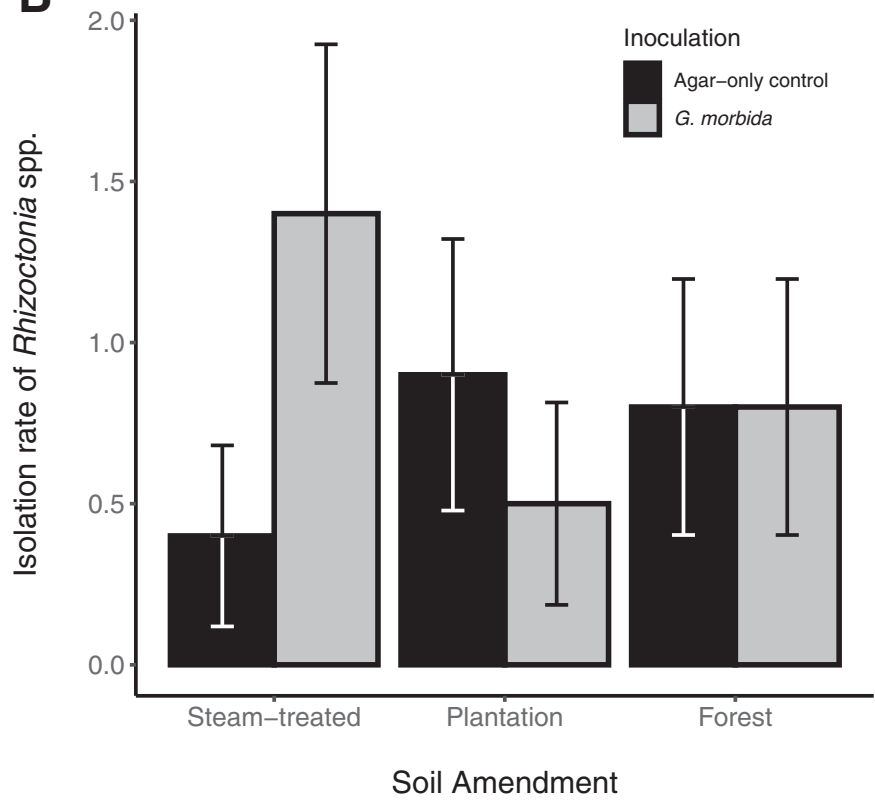

Fig. 4. Isolation rates (mean \pm overdispersion-adjusted standard error number of colonies per plant) of A, Fusarium spp. and B, Rhizoctonia spp. from roots of walnut seedlings after being amended with steam-treated, plantation, and forest soil and inoculated with Geosmithia morbida or agar-only control.

A

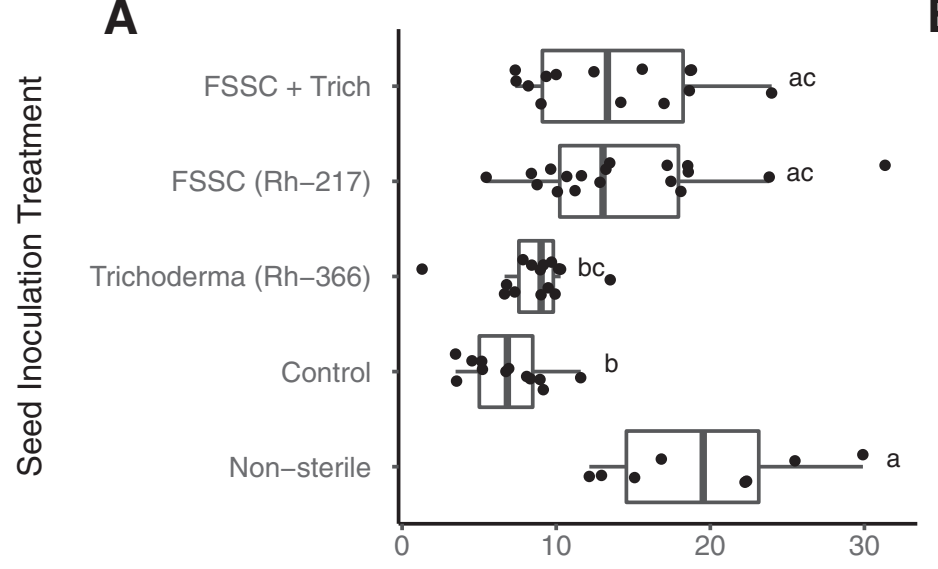

Necrotic Area $\left(\mathrm{mm}^{2}\right)$
B

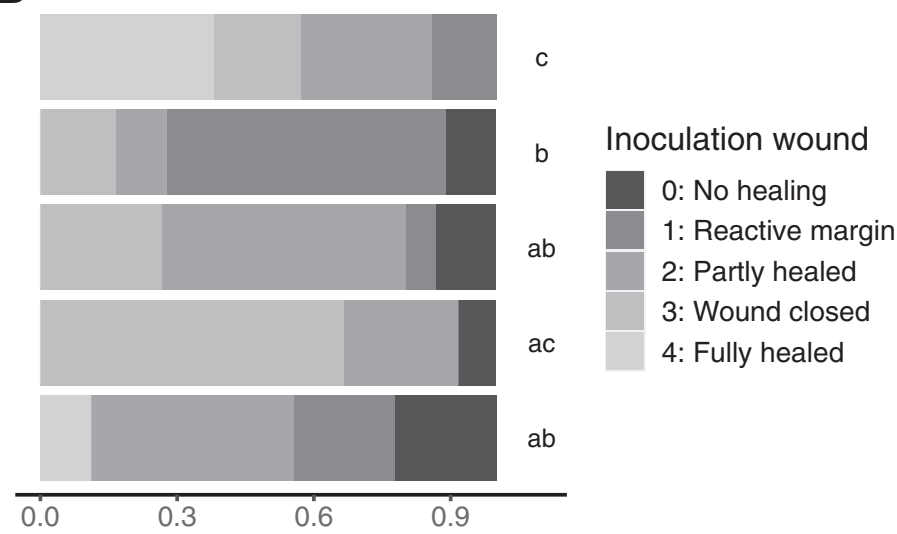

Proportion of replicates

Fig. 5. A, Necrotic area and B, healing of cankers caused by Geosmithia morbida in seedlings grown in untreated potting mix (Nonsterile) or inoculated with filtrate only (Control), Trichoderma sp. isolate Rh-366, Fusarium solani species complex (FSSC) sp. isolate Rh-217, or both Rh-366 and Rh-217 together (FSSC + Trich). Different letters in plot A denote significant Tukey-adjusted contrasts from a Box-Cox $(\lambda=0.4)$ transformed linear model. Outliers not shown. Different letters in plot B denote significant unadjusted contrasts from a log-link proportional odds model. 
99.8 Rh515 (Roots, Non-sterile MW300978 \& MW456961)

NRRL 22938 FSSC 3+4-9

MW300981 \& MW456964)

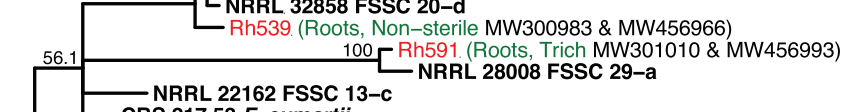

58.2 CBS 217.53 F. eumartii

61.8 LRR NRRL 31169 FSSC 25-a

NRRL 46707 FSSC $35-a$

Rh624* (Roots, Trich MW301034 \& MW457017)

Rh623* (Roots, Trich MW301033 \& MW457016)

Rh622* (Roots, FSSC + Trich MW301032 \& MW457015)

Rh621* (Roots, FSSC + Trich MW301031 \& MW457014)

Rh620* (Roots, FSSC MW301030 \& MW457013)

Rh619* (Roots, FSSC MW301029 \& MW457012)

Rh618* (Roots, FSSC MW301028 \& MW457011)

Rh617* (Roots, FSSC MW301027 \& MW457010)

Rh615* (Roots, FSSC + Trich MW301026 \& MW457009)

Rh61 ** (Roots, FSSC + Trich MW301026 \& MW457009)

Rh612* (Roots, Trich MW301024 \& MW457007)

Rh612* (Roots, Trich MW301024 \& MW457007)

Rh610* (Roots, Control MW301022 \& MW457005)

Rh69* (Roots, Control MW301021 \& MW457004)

Rh608* (Roots, FSSC MW301020 \& MW457003)

Rh603* (Sols,

Rh602* (Soil, Control MW301016 \& MW456999)

Rh601* (Soil, FSSC + Trich MW301015 \& MW456998)

R60 * (Soi, FSSC + Trich MW301014 \& MW456998)

Rh60*

Rh596* (Roots, Control MW301013 \& MW456996)

(

Rh589* (Roots, FSSC + Trich MW301009 \& MW456992)

Rich

Rh58* (Roots, FSSC MW301007 \& MW456990)

Rh50* (Roots, FSSC MW301006 \& MW456989)

(

(

(1)

Ris

96.4 Rh5 * (Roots, FSSC + Tich MW300998 \& MW456981)

(

Rh565* (Roots, Control MW300994 \& MW456977)

Rh564* (Roots, Control MW300993 \& MW456976)

Rh563* (Roots, FSSC MW300992 \& MW456975)

Rh562* (Roots, FSSC + Trich MW300991 \& MW456974)

Rh561* (Root, FSSC + Trich MW300990 \& MW456973)

Rh560* (Roots, FSSC + Trich MW300989 \& MW456972)

Rh550* (Roots, FSSC MW300986 \& MW456969)

Rh548* (Roots, FSSC MW300985 \& MW456968)

Rh545 (Roots, FSSC MW300984 \& MW456967)

Rh532* (Roots, Non-sterile MW300982 \& MW456965)

Rh518* (Roots, FSSC MW300980 \& MW456963)

Rh516* (Roots, Non-sterile MW300979 \& MW456962)

Rh514* (Roots, Control MW300977 \& MW456960)

Rh513* (Roots, FSSC + Trich MW300976 \& MW456959)

Rh509* (Roots, FSSC + Trich MW300975 \& MW456958)

Rh508* (Roots, FSSC MW300974 \& MW456957)

Rh501* (Soil, Trich MW300972 \& MW456955)

Rh217* (Roots, Experiment 1 MW300971 \& MW456954)

Lily73* (Canker, FSSC + Trich MW300970 \& MW456953)

Lily72* (Canker, FSSC + Trich MW300969 \& MW456952

Lily71* (Canker, FSSC + Trich MW300968 \& MW456951)

Lily69* (Canker, FSSC MW300966 \& MW456949)

Lily68 (Canker, FSSC MW300965 \& MW456948)

Lily67* (Canker, FSSC + Trich MW300964 \& MW456947)

Lily66* (Canker, FSSC + Trich MW300963 \& MW456946)

Lily65* (Canker, FSSC + Trich MW300962 \& MW456945)

Lily64* (Canker, Control MW300961 \& MW456944)

Lily63* (Canker, Non-sterile MW300960 \& MW456943)

Lily62* (Canker, Non-sterile MW300959 \& MW456942)

Lily61* (Canker, Non-sterile MW300958 \& MW456941)

Lily70 (Canker, FSSC MW300967 \& MW456950)

100 Rh574 (Soll, Control MW301002 \& MW456985) \& MW456982

Rh559 (Roots, Control MW300988 \& MW456971)

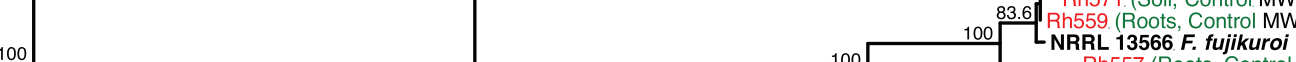

100 Rh557 (Roots, Control MW300987 \& MW456970) Rh594 (Roots, Control MW301011 \& MW456994)

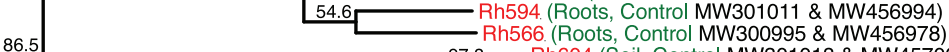
27 Rh566 (Roots, Control MW300995 \& MW456978)

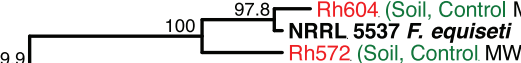

99.9 Rh572 (Soil, Control MW301000 \& MW456983)

99.3 — Rh504 (Soil, Control MW300973 \& MW456956)

- NRRL 25474 F. sporotrichioides

NRRL 20715 F. dimerum

$\begin{array}{llll}1 & 100 & 200 & 300\end{array}$

Fig. 6. Likelihood-based multilocus placement of Fusarium spp. encountered while reisolating Fusarium solani species complex (FSSC) sp. isolate Rh-217 from endophyte inoculation experiment. Majority-rules consensus (1,000 bootstrap RAxML trees, GTR+CAT model, all nodes $>50 \%$ ) of concatenated internal transcribed spacer (ITS) and translation elongation factor 1- $\alpha$ (TEF-1a) alignments (561 alignment patterns) from Fusarium cultures. Branch lengths proportional to substitutions per site estimated via GTR+I+G in PAUP; node values denote percent of supporting bootstrap replicates. Vouchers isolated in this study are given in red along with isolation source (roots, cankers, or soil), and treatment code (Nonsterile, Control, Trichoderma [Trich], FSSC, and FSSC + Trichoderma) in dark green, followed by GenBank accession numbers (ITS and TEF-1) in black. Isolates from the Fusarium multilocus sequence typing database (O'Donnell et al. 2012) are given in bold. An asterisk (*) indicates isolates with identical ITS+TEF-1a sequences. 


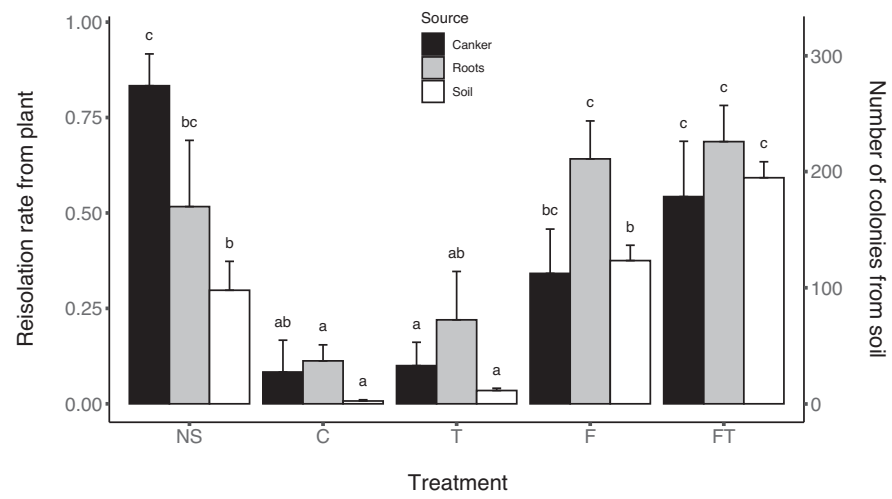

Fig. 7. Reisolation rates (mean + adjusted standard error number of colonies per plant) of Fusarium solani species complex (FSSC) sp. isolate Rh-217 from cankers, roots (left axis), and soil (right axis) of walnut seedlings grown in untreated potting mix (NS) or in sterilized potting mix inoculated with filtrate only (C), Trichoderma asperellum Rh-366 (T), FSSC Rh-217 (F), or both Rh-217 and Rh-366 together (FT). Counts are of colonies whose morphological vouchers had $100 \%$ identity to the internal transcribed spacer plus translation elongation factor $1-\alpha$ sequence of FSSC sp. isolate Rh-217. Groups with different letters had significantly different reisolation rates within cankers, roots, or soil.

(Montecchio et al. 2015). In total, 59 isolates from cankers $(n=$ $11)$, roots $(n=40)$, and soil $(n=8)$ were $100 \%$ identical to FSSC isolate Rh-217 across the merged ITS+TEF1 alignment (Figs. 6 and 7). Fungi that were classified to the same morphospecies as these molecular vouchers, which we inferred to be FSSC sp. isolate Rh-217, were isolated at significantly different rates across treatments (Fig. 7) $(P \leq 0.001 ; d f=4)$. FSSC sp. isolate Rh-217 was reisolated more frequently from FSSC, FSSC + Trichoderma, and nonsterile treatments than from the control seedlings $(P<0.05)$. Trichoderma sp. isolate Rh-366 (GenBank accessions MW301039 and MW301040) was also reisolated but from very few plants.

Reisolation rates of $G$. morbida IN-66 from cankers differed significantly among treatments $(P=0.028 ; d f=4)$. Geosmithia morbida was isolated from the Trichoderma treatment $6.0 \pm 5.2$ times more frequently than the nonsterile treatment $(P=0.037), 2.1 \pm 0.9$ times more frequently than the FSSC treatment $(P=0.056)$, and $3.2 \pm 1.5$ times more frequently than the FSSC + Trichoderma treatment $(P=0.010)$.

\section{DISCUSSION}

Our findings support the hypothesis that the soil microbiome in natural forests can indirectly enhance the resistance of walnut trees to G. morbida. Plant-mediated feedback between primary aboveground pathogens and above- and belowground latent endophytic pathogens may predispose J. nigra seedlings to successful infection by $G$. morbida, leading to larger cankers around inoculation points. These findings are consistent with previous work which also demonstrated that the microbiome associated with aboveground woody tissues of mature eastern black walnut trees shifts in response to colonization by G. morbida (Onufrak et al. 2020). This feedback appears to be dampened by forest soil microbiota, protecting seedlings from disease. In particular, forest soil microbiota suppressed the abundance of cosmopolitan FSSC spp. Suppression of Fusarium pathogens with fungi from forest soil has been demonstrated in annual crops and conifer nursery systems (Chakravarty and Hwang 1991; Chakravarty and Unestam 1987; Chakravarty et al. 1991; Ridout and Newcombe 2016; Sylvia and Sinclair 1983).
With live soil amendment and endophyte inoculation experiments, we observed plant-mediated positive feedback between G. morbida and FSSC spp. Fungi in the FSSC have the ability to asymptomatically colonize root cortical cells of seedlings during a latent period before producing phytotoxins and switching to a necrotrophic habit (Bacon and Yates 2006; Swett et al. 2016). By contrast, G. morbida caused immediate necrosis in our experiments, and likely exploits oxidative defenses to feed on dead tissue (Govrin and Levine 2000). Thus, prior colonization by Fusarium spp. could have led to a physiological state of hypersensitivity or systemic induced susceptibility to G. morbida (Eyles et al. 2010; Plett and Martin 2018). However, our findings also suggest that interactions between Fusarium spp., J. nigra, and G. morbida are further regulated by the entire soil microbiome or substrate availability and suitability. This dependence of aboveground-belowground feedback on the soil microbiome and substrate is similar to the findings of van Gils et al. (2017) for aphids and Rhizoctonia spp. in an annual crop. When soil amendments were steam-treated in our study, aboveground inoculation with G. morbida inhibited belowground colonization by Fusarium spp. and promoted Rhizoctonia spp. In contrast, in seedings grown with plantation soil amendments, Fusarium was promoted and Rhizoctonia was suppressed by aboveground G. morbida inoculation; this feedback was suppressed by the addition of live forest soil. Thus, forest soil amendments appear to impart greater resilience to the perturbation of the rhizosphere caused by aboveground pathogen infection.

In our study, legacy effects of forest management in the soil microbiome may have contributed to the susceptibility of seedlings to G. morbida. Plantation trees are grown at high density and low diversity and are likely to experience heavy pressure from insects and physical damage (Coyle et al. 2005). Therefore, plantations in our study may have been enriched in the rhizosphere with host-adapted biotrophs or hemibiotrophs like Fusarium spp. that heightened host susceptibility to necrotrophic attack by G. morbida. Similarly, the annual plant Arabidopsis thaliana accumulated necrotrophs when grown in soils conditioned by plants infected aboveground by a biotroph (Yuan et al. 2018). If woody plants similarly condition their soils in response to attack by bark beetles and their necrotrophic fungal symbionts, a corresponding accumulation of specialist biotrophs in the rhizosphere could have important ecological implications for forest regeneration and diversity. The microbiome of seedlings grown in fumigated nursery beds possibly induced a heightened state of defense against Fusarium spp. but heightened susceptibility to necrotrophs such as Rhizoctonia spp. and G. morbida that exploit oxidative defenses (Foley et al. 2016; Govrin and Levine 2000). This physiological state could have been reversed by $G$. morbida inoculation or forest soil amendments, leading to responses in belowground community structure.

Despite the importance of phytobiomes in plant disease, their interactions with forest management practices and forest health are seldom explored (Busby et al. 2017). Here, we provide evidence suggesting that infection with FSSC spp., high host density, and low genetic diversity in plantations are predisposing factors that could increase the susceptibility of J. nigra to G. morbida, the primary fungal symbiont of $P$. juglandis, which together cause TCD. Our findings are consistent with the hypotheses that (i) some endophytes function as latent pathogens and (ii) plant diseases of forest trees might be attributed to consortia of multiple pests and pathogens or disease synergists and secondary pathogens (Busby et al. 2016; Carroll 1988; Lamichhane and Venturi 2015; Mazzola 1998). Disease synergists such as FSSC spp. are abundant both as fungal endophytes and in the natural environment, and frequently encountered as secondary pathogens in TCD-symptomatic trees (Kasson 
et al. 2014; Tisserat et al. 2009). However, they might be less abundant in forests (Ridout and Newcombe 2016).

Intensively managed forest agroecosystems might be predisposed to disease, in part due to accumulation of opportunistic pathogens such as Fusarium spp. In this study, FSSC spp. could have come from potting mix, from the fumigated nursery beds, or as a vertically transmitted endophytes in seed (Belisario et al. 2002; Dalling et al. 2020; Liu et al. 2010; Newcombe et al. 2018). Management scenarios that make use of these vectors and substrates could favor Fusarium spp. as a first colonizer in the endosphere of seedlings and young trees. If Fusarium spp. then persist as dominant members of the microbiome, cultivation or intensive orchard seed production of J. nigra seedlings may predispose trees to TCD (Ridout et al. 2019). The extent to which first colonizers such as FSSC Rh-217, which we used to inoculate germinating seed, persist in the tissues of mature trees and propagate in plant communities remains poorly understood and is a critical knowledge gap in the field of forest pathology (Newcombe 2011). Further study should aim to build an understanding of the influence that common soil and seeddwelling pathogens in orchards and nurseries have on the productivity of hardwood plantations and their susceptibility to disease.

In addition to possible enrichment with secondary pathogens, soil microbiomes in urban forests and plantations, like those in the nonnative range of $J$. nigra, are possibly depauperate in beneficial microbiota relative to forests (Onufrak et al. 2020). Our culturebased study design did not permit the detection of arbuscular mycorrhizal fungi (AMF), which cannot be grown in nutrient media, nor did we investigate plant-growth-promoting rhizobacteria (PGPR). AMF and PGPR could have induced resistance to G. morbida (Clear and Hom 2019). AMF might have been more abundant or beneficial to black walnut in forest soils than plantation soils, and could have suppressed Fusarium spp. in roots (Eke et al. 2016; Ponder 1979). Zopfiella spp., which were predominantly found in forest soil treatments, have been identified as potential biological control agents and implicated in suppression of soilborne disease (Liu et al. 2019; Zhao et al. 2018). In contrast, Trichoderma $\mathrm{Rh}-366$, which is related to a putative biological control for other Fusarium spp. (F. oxysporum and F. graminearum) (He et al. 2019; Patel and Saraf 2017), did not decrease canker size or the amount of infection by FSSC Rh-217 when coinoculated. Nevertheless, Trichoderma sp. isolate $\mathrm{Rh}-366$ stimulated more advanced healing of inoculation wounds, which is consistent with findings in other pathosystems, including the application of Trichoderma spp. to protect seedlings from aboveground herbivores (Pineda et al. 2017). Further research could investigate whether Trichoderma spp. are effective as seed pretreatments to block subsequent Fusarium colonization (Ridout et al. 2019).

Advanced knowledge of the contextual factors of nursery, plantation, and urban forest management and their collective effect on the host microbiome will have important implications for the management of pests and pathogens. Our findings suggest that the microbiome of long-lived hosts in mixed hardwood stands without groundcover management might protect trees from disease by suppressing negative soil feedbacks (Pineda et al. 2017; Schlatter et al. 2017). Therefore, further study should be conducted to explore the role of soil feedbacks in TCD and other tree diseases caused by pathogens and insect vectors. Foresters and funding agencies may consider that a better understanding of microbial legacy effects of prior land use and nursery practices, higher species and genetic diversity, and holistic soil, groundcover, and forest plantation management will lead to improvements in yield, quality, and resilience to biotic disturbance.

Supporting material. Data, sequences, and R and mothur scripts are available in a downloadable repository at https://github.com/
readingradio/WilliamsGinzel.JnGmFusarium.2021. Voucher cultures of G. morbida isolate IN-66, FSSC sp. isolate Rh-217, and Trichoderma sp. isolate Rh-366 used for inoculations, along with representatives of the most commonly isolated OTUs, are held in long-term storage collections at Purdue University, Department of Forestry and Natural Resources and can be made available upon request.

\section{ACKNOWLEDGMENTS}

We thank J. McKenna, M. C. Aime, M. Coggeshall, B. Beheler, S. Cambron, J. Couture, S. Cho, A. Howes, N. Cazzaniga, T. Ailes, C. Prechtel, and S. Paul, without whose assistance and resource sharing this work would not have been possible; and K. Tobin, M. Ethington, C. Smallwood, and Đ. Hadžiabdić for helpful feedback on the manuscript.

\section{LITERATURE CITED}

Altschul, S. 1990. Basic Local Alignment Search Tool. J. Mol. Biol. 215:403-410. Arnold, A. E., Maynard, Z., Gilbert, G. S., Coley, P. D., and Kursar, T. A. 2000. Are tropical fungal endophytes hyperdiverse? Ecol. Lett. 3:267-274.

Aukema, B. H., Zhu, J., Møller, J., Rasmussen, J. G., and Raffa, K. F. 2010. Predisposition to bark beetle attack by root herbivores and associated pathogens: Roles in forest decline, gap formation, and persistence of endemic bark beetle populations. For. Ecol. Manage. 259:374-382.

Bacon, C. W., and Yates, I. E. 2006. Endophytic root colonization by Fusarium species: Histology, plant interactions, and toxicity. Pages 133152 in: Microbial Root Endophytes. B. Schultz, B. Boyle, and T. Sieber, eds. Springer, Berlin, Heidelberg, Germany.

Belisario, A., Maccaroni, M., Corazza, L., Balmas, V., and Valier, A. 2002. Occurrence and etiology of brown apical necrosis on Persian (English) walnut fruit. Plant Dis. 86:599-602.

Bennett, J. A., and Klironomos, J. 2019. Mechanisms of plant-soil feedback: Interactions among biotic and abiotic drivers. New Phytol. 222:91-96.

Browne, G., Lee, R., Mclaughlin, S., Connell, J., Schneider, S., and Bulluck, R. 2006. Investigating associations between culturable soilborne fungi and replant disease of stone fruits in California. Phytopathology 96:S166.

Busby, P. E., Ridout, M., and Newcombe, G. 2016. Fungal endophytes: Modifiers of plant disease. Plant Mol. Biol. 90:645-655.

Busby, P. E., Soman, C., Wagner, M. R., Friesen, M. L., Kremer, J., Bennett, A., Morsy, M., Eisen, J. A., Leach, J. E., and Dangl, J. L. 2017. Research priorities for harnessing plant microbiomes in sustainable agriculture. PLoS Biol. 15:e2001793.

Carroll, G. 1988. Fungal endophytes in stems and leaves: From latent pathogen to mutualistic symbiont. Ecology 69:2-9.

Castellá, G., Bragulat, M. R., Rubiales, M. V., and Cabañes, F. J. 1997. Malachite green agar, a new selective medium for Fusarium spp. Mycopathologia 137:173-178.

Cesarano, G., Zotti, M., Antignani, V., Marra, R., Scala, F., and Bonanomi, G. 2017. Soil sickness and negative plant-soil feedback: A reappraisal of hypotheses. J. Plant Pathol. 99:545-570.

Chakravarty, C., Peterson, R. L., and Ellis, B. E. 1991. Interaction between the ectomycorrhizal fungus Paxillus involutus, damping-off fungi and Pinus resinosa seedlings. J. Phytopathol. 132:207-218.

Chakravarty, P., and Hwang, S. F. 1991. Effect of an ectomycorrhizal fungus, Laccaria laccata, on Fusarium damping-off in Pinus banksiana seedlings. Eur. J. Forest Pathol. 21:97-106.

Chakravarty, P., and Unestam, T. 1987. Differential influence of ectomycorrhizae on plant growth and disease resistance in Pinus sylvestris seedlings. J. Phytopathol. 120:104-120.

Clear, M. R., and Hom, E. F. Y. 2019. The evolution of symbiotic plantmicrobe signalling. Annu. Plant Rev. Online 2.

Coyle, D. R., Nebeker, T. E., Hart, E. R., and Mattson, W. J. 2005. Biology and management of insect pests in North American intensively managed hardwood forest systems. Annu. Rev. Entomol. 50:1-29.

Dalling, J. W., Davis, A. S., Arnold, A. E., Sarmiento, C., and Zalamea, P.-C. 2020. Extending plant defense theory to seeds. Annu. Rev. Ecol. Evol. Syst. 51:123-141.

Durrant, W. E., and Dong, X. 2004. Systemic acquired resistance. Annu. Rev. Phytopathol. 42:185-209. 
Edgar, R. C. 2004. MUSCLE: A multiple sequence alignment method with reduced time and space complexity. BMC Bioinf. 5:113.

Eke, P., Chatue, G., Wakam, L. N., Kouipou, R. M. T., Fokou, P. V. T., and Boyom, F. F. 2016. Mycorrhiza consortia suppress the fusarium root rot (Fusarium solani f. sp. Phaseoli) in common bean (Phaseolus vulgaris L.). Biol. Control 103:240-250.

Ennos, R. A. 2015. Resilience of forests to pathogens: An evolutionary ecology perspective. Forestry 88:41-52.

Eyles, A., Bonello, P., Ganley, R., and Mohammed, C. 2010. Induced resistance to pests and pathogens in trees. New Phytol. 185:893-908.

Foley, R. C., Kidd, B. N., Hane, J. K., Anderson, J. P., and Singh, K. B. 2016. Reactive oxygen species play a role in the infection of the necrotrophic fungi, Rhizoctonia solani in wheat. PLoS One 11:e0152548.

Fox, J., and Weisberg, S. 2019. An R Companion to Applied Regression. Sage, Thousand Oaks, CA, U.S.A.

Gazis, R., Poplawski, L., Klingeman, W., Boggess, S. L., Trigiano, R. N., Graves, A. D., Seybold, S. J., and Hadziabdic, D. 2018. Mycobiota associated with insect galleries in walnut with thousand cankers disease reveals a potential natural enemy against Geosmithia morbida. Fungal Biol. 122:241-253.

Geiser, D. M., del Mar Jiménez-Gasco, M., Kang, S., Makalowska, I., Veeraraghavan, N., Ward, T. J., Zhang, N., Kuldau, G. A., and O'Donnell, K. 2004. FUSARIUM-ID v. 1.0: A DNA sequence database for identifying Fusarium. Eur. J. Plant Pathol. 110:473-479.

Govrin, E. M., and Levine, A. 2000. The hypersensitive response facilitates plant infection by the necrotrophic pathogen Botrytis cinerea. Curr. Biol. 10:751-757.

He, A., Liu, J., Wang, X., Zhang, Q., Song, W., and Chen, J. 2019. Soil application of Trichoderma asperellum GDFS1009 granules promotes growth and resistance to Fusarium graminearum in maize. J. Integr. Agric. 18:599-606.

Hertert, H. D., Miller, D. L., and Partridge, A. D. 1975. Interaction of bark beetles (Coleoptera: Scolytidae) and root-rot pathogens of grand-fir in northern Idaho. Can. Entomol. 107:899-904.

Jactel, H., Moreira, M., and Castageneyrol, B. 2021. Tree diversity and forest resistance to insect pests: Patterns, mechanisms, and prospects. Annu. Rev. Entomol. 66:277-296.

Jeffers, S. N., and Martin, S. B. 1986. Comparison of two media selective for Phytophthora and Pythium species. Plant Dis. 70:1038-1043.

Jung, S. C., Martinez-Medina, A., Lopez-Raez, J. A., and Pozo, M. J. 2012. Mycorrhiza-induced resistance and priming of plant defenses. J. Chem. Ecol. 38:651-664.

Juzwik, J., Banik, M. T., Reed, S. E., English, J. T., and Ginzel, M. D. 2015. Geosmithia morbida Found on Weevil Species Stenomimus pallidus in Indiana. Plant Health Prog. 16:7-10.

Juzwik, J., Moore, M., Williams, G., and Ginzel, M. 2020. Assessment and etiology of thousand cankers disease within the native range of black walnut (Juglans nigra). Pages 169-178 in: Forest Health Monitoring: National Status, Trends, and Analysis 2019. Gen. Tech. Rep. SRS-250. K. M. Potter and B. L. Conkling, eds. United States Department of Agriculture, Forest Service, Southern Research Station, Asheville, NC, U.S.A.

Kaplan, I., Pineda, A., and Bezemer, M. 2018. Application and Theory of Plant-Soil Feedbacks on Aboveground Herbivores. Pages 319-343 in: Aboveground-Belowground Community Ecology. T. Ohgushi, S. Wurst, and S. N. Johnson, eds. Springer International Publishing, Cham, Switzerland.

Kasson, M. T., Griffin, G. J., Geiser, D. M., O’Neal, E. S., Juzwik, J., Reed, S., Tisserat, N., Turcotte, R. M., Martin, D. K., Davis, D. D., Fenstermacher, K. A., and O'Donnell, K. 2014. New perspectives on thousand cankers disease of walnut. Phytopathology 104:S3.148.

Klein, R. M., and Perkins, T. D. 1987. Cascades of causes and effects of forest decline. Ambio 16:86-93.

Klironomos, J. N. 2002. Feedback with soil biota contributes to plant rarity and invasiveness in communities. Nature 417:67-70.

Lamichhane, J. R., and Venturi, V. 2015. Synergisms between microbial pathogens in plant disease complexes: A growing trend. Front. Plant Sci. 6:385.

Lauritzen, J. E. 2018. Characterization of Black Walnut Genotypes for Resistance to Thousand Cankers Disease, Frost Hardiness and Other Desirable Horticultural Traits. Ph.D. thesis, Utah State University, Logan, UT, U.S.A.

Lee, S. B., and Taylor, J. W. 1990. Isolation of DNA from fungal mycelia and single spores. Pages 282-287 in: PCR Protocols: A Guide to Methods and Applications. M. Innis, D. Gelfand, J. Sninsky, and T. White, eds. Academic Press, New York, NY, U.S.A.

Leslie, J. F., and Summerell, B. A. 2008. The Fusarium Laboratory Manual. John Wiley \& Sons, Denver, CO, U.S.A.

Liu, B., Sun, J., Rivard, C., Welker, R., and Louws, F. 2010. Comparison of fumigation, mustard meal amendments and grafting on bulk soil microbial communities in tomato fields. Phytopathology 100:S73.

Liu, L., Huang, X., Zhao, J., Zhang, J., and Cai, Z. 2019. Characterizing the key agents in a disease-suppressed soil managed by reductive soil disinfestation. Appl. Environ. Microbiol. 85:e02992-18.

Maddison, D. R., and Maddison, W. P. 2019b. Chromaseq: A Mesquite package for analyzing sequence chromatograms. Version 1.51. http:// mesquiteproject.org/packages/chromaseq/

Maddison, W. P., and Maddison, D. R. 2019a. Mesquite: A modular system for evolutionary analysis. Version 3.61. http://www.mesquiteproject.org/

Manion, P. D. 1981. Tree Disease Concepts. Prentice-Hall, Inc., Englewood Cliffs, NJ, U.S.A.

Mazzola, M. 1998. Elucidation of the microbial complex having a causal role in the development of apple replant disease in Washington. Phytopathology 88:930-938.

McFadden, D. 1974. The measurement of urban travel demand. J. Public Econ. 3:303-328.

McKenry, M. 1999. The Replant Problem and its Management. Catalina Publishing, Fresno, CA, U.S.A.

Mielke, M. E., and Ostry, M. E. 2004. Diseases of intensively managed eastern black walnut. Pages 110-113 in: Proc. 6th Walnut Counc. Res. Symp. Gen. Tech. Rep. NC-243. C. H. Michler, P. M. Pijut, J. W. Van Sambeek, M. V. Coggeshall, J. Seifert, K. Woeste, R. Overton, and F. Ponder, eds. United States Department of Agriculture, Forest Service, North Central Research Station, St. Paul, MN, U.S.A.

Montecchio, L., Faccoli, M., Short, D. P. G., Fanchin, G., Geiser, D. M., and Kasson, M. T. 2015. First report of Fusarium solani phylogenetic species 25 associated with early stages of thousand cankers disease on Juglans nigra and Juglans regia in Italy. Plant Dis. 99:1183.

Nakagawa, S., Johnson, P. C. D., and Schielzeth, H. 2017. The coefficient of determination $R^{2}$ and intra-class correlation coefficient from generalized linear mixed-effects models revisited and expanded. J. R. Soc. Interface 14:20170213

Newcombe, G. 2011. Endophytes in forest management: Four challenges. Pages 251-262 in: Endophytes of Forest Trees. A. M. Pirttilä and A. C. Frank, eds. Forestry Sciences, vol. 80. Springer, Dordrecht, The Netherlands.

Newcombe, G., Harding, A., Ridout, M., and Busby, P. E. 2018. A hypothetical bottleneck in the plant microbiome. Front. Microbiol. 9:1645.

Newhouse, J. R., and Hunter, B. B. 1983. Selective media for recovery of Cylindrocladium and Fusarium species from roots and stems of tree seedlings. Mycologia 75:228-233.

Nilsson, R. H., Larsson, K.-H., Taylor, A. F. S., Bengtsson-Palme, J., Jeppesen, T. S., Schigel, D., Kennedy, P., Picard, K., Glöckner, F. O., Tedersoo, L., Saar, I., Kõljalg, U., and Abarenkov, K. 2019. The UNITE database for molecular identification of fungi: Handling dark taxa and parallel taxonomic classifications. Nucleic Acids Res. 47:D259-D264.

O'Donnell, K., Al-Hatmi, A. M. S., Aoki, T., Brankovics, B., Cano-Lira, J. F., Coleman, J. J., de Hoog, G. S., Di Pietro, A., Frandsen, R. J. N., Geiser, D. M., Gibas, C. F. C., Guarro, J., Kim, H.-S., Kistler, H. C., Laraba, I., Leslie, J. F., López-Berges, M. S., Lysøe, E., Meis, J. F., Monod, M., Proctor, R. H., Rep, M., Ruiz-Roldán, C., Šišić, A., Stajich, J. E., Steenkamp, E. T., Summerell, B. A., van der Lee, T. A. J., van Diepeningen, A. D., Verweij, P. E., Waalwijk, C., Ward, T. J., Wickes, B. L., Wiederhold, N. P., Wingfield, M. J., Zhang, N., and Zhang, S. X. 2020. No to Neocosmospora: Phylogenomic and practical reasons for continued inclusion of the Fusarium solani species complex in the genus. MSphere 5:e00810-20.

O’Donnell, K., Humber, R. A., Geiser, D. M., Kang, S., Park, B., Robert, V. A. R. G., Crous, P. W., Johnston, P. R., Aoki, T., Rooney, A. P., and Rehner, S. A. 2012. Phylogenetic diversity of insecticolous fusaria inferred from multilocus DNA sequence data and their molecular identification via FUSARIUM-ID and Fusarium MLST. Mycologia 104:427-445.

O’Donnell, K., Kistler, H. C., Cigelnik, E., and Ploetz, R. C. 1998. Multiple evolutionary origins of the fungus causing Panama disease of banana: Concordant evidence from nuclear and mitochondrial gene genealogies. Proc. Natl. Acad. Sci. U.S.A. 95:2044-2049.

Oksanen, J., Blanchet, F. G., Friendly, M., Kindt, R., Legendre, P., McGlinn, D., Minchin, P. R., O’Hara, R. B., Simpson, G. L., Solymos, P., Stevens, 
M. H. H., Szoecs, E., and Wagner, H. 2018. vegan: Community Ecology Package. https://cran.r-project.org/web/packages/vegan/index.html

Onufrak, A. J., Williams, G. M., Klingeman, W. E., Cregger, M. A., Klingeman, D. M., DeBruyn, J. M., Ginzel, M. D., and Hadziabdic, D. 2020. Regional differences in the structure of Juglans nigra phytobiome reflect geographical differences in thousand cankers disease severity. Phytobiomes J. 4:388-404.

Patel, S., and Saraf, M. 2017. Biocontrol efficacy of Trichoderma asperellum MSST against tomato wilting by Fusarium oxysporum f. sp. lycopersici. Arch. Phytopathol. Plant Prot. 50:228-238.

Pineda, A., Kaplan, I., and Bezemer, T. M. 2017. Steering soil microbiomes to suppress aboveground insect pests. Trends Plant Sci. 22:770-778.

Plett, J. M., and Martin, F. M. 2018. Know your enemy, embrace your friend: Using omics to understand how plants respond differently to pathogenic and mutualistic microorganisms. Plant J. 93:729-746.

Ploetz, R. C., Hulcr, J., Wingfield, M. J., and de Beer, Z. W. 2013. Destructive tree diseases associated with ambrosia and bark beetles: Black swan events in tree pathology? Plant Dis. 97:856-872.

Ponder, F. 1979. Soil structure and mycorhizae encourage black walnut growth on old fields. Res. Note NC-249. United States Department of Agriculture, Forest Service, North Central Forest Experimental Station, St. Paul, MN, U.S.A.

Porras-Alfaro, A., and Bayman, P. 2011. Hidden fungi, emergent properties: Endophytes and microbiomes. Annu. Rev. Phytopathol. 49:291-315.

R Core Team. 2019. R: A Language and Environment for Statistical Computing. https://cran.r-project.org

Ridout, M., Houbraken, J., and Newcombe, G. 2017. Xerotolerance of Penicillium and Phialocephala fungi, dominant taxa of fine lateral roots of woody plants in the intermountain Pacific Northwest, USA. Rhizosphere 4:94-103.

Ridout, M., and Newcombe, G. 2016. Disease suppression in winter wheat from novel symbiosis with forest fungi. Fungal Ecol. 20:40-48.

Ridout, M. E., Schroeder, K. L., Hunter, S. S., Styer, J., and Newcombe, G. 2019. Priority effects of wheat seed endophytes on a rhizosphere symbiosis. Symbiosis 78:19-31.

Schlatter, D., Kinkel, L., Thomashow, L., Weller, D., and Paulitz, T. 2017. Disease suppressive soils: New insights from the soil microbiome. Phytopathology 107:1284-1297.

Schloss, P. D., Westcott, S. L., Ryabin, T., Hall, J. R., Hartmann, M., Hollister, E. B., Lesniewski, R. A., Oakley, B. B., Parks, D. H., Robinson, C. J., Sahl, J. W., Stres, B., Thallinger, G. G., Van Horn, D. J., and Weber, C. F. 2009. Introducing mothur: Open-source, platform-independent, community-supported software for describing and comparing microbial communities. Appl. Environ. Microbiol. 75:7537-7541.

Schmidt, L., Bhat, R., and Browne, G. 2010. Culture-independent examination of microbial community shifts associated with replant disease of almond. Phytopathology 100:S115.

Seybold, S. J., Klingeman, W. E., Hishinuma, S. M., Coleman, T. W., and Graves, A. D. 2019. Status and impact of walnut twig beetle in urban forest, orchard, and native forest ecosystems. J. For. 117:152-163.

Sitz, R. A., Luna, E. K., caballero, J. I., Tisserat, N. A., Cranshaw, W. S., and Stewart, J. E. 2017. Virulence of genetically distinct Geosmithia morbida isolates to black walnut and their response to coinoculation with Fusarium solani. Plant Dis. 101:116-120.

Stamatakis, A. 2014. RAxML version 8: A tool for phylogenetic analysis and post-analysis of large phylogenies. Bioinformatics 30:1312-1313.
Summerell, B. A. 2019. Resolving Fusarium: Current status of the genus. Annu. Rev. Phytopathol. 57:323-339.

Swett, C. L., Kirkpatrick, S. C., and Gordon, T. R. 2016. Evidence for a hemibiotrophic association of the pitch canker pathogen Fusarium circinatum with Pinus radiata. Plant Dis. 100:79-84.

Swofford, D. L. 2003. PAUP*. Phylogenetic Analysis Using Parsimony (*and Other Methods). Version 4. Sinauer Associates, Sunderland, MA, U.S.A.

Sylvia, D. M., and Sinclair, W. A. 1983. Suppressive influence of Laccaria laccata on Fusarium oxysporum and on Douglas-fir seedlings. Phytopathology 73:384-389.

Tainter, F. H., and Baker, F. A. 1996. Principles of Forest Pathology. John Wiley \& Sons, New York, NY, U.S.A.

Tello, M.-L., Tomalak, M., Siwecki, R., Gáper, J., Motta, E., and MateoSagasta, E. 2005. Biotic urban growing conditions-Threats, pests and diseases. Pages 325-365 in: Urban Forests and Trees. C. Konijnendijk, K. Nilsson, T. Randrup, and J. Schipperijn, eds. Springer, Berlin, Heidelberg, Germany.

Tisserat, N., Cranshaw, W., Leatherman, D., Utley, C., and Alexander, K. 2009. Black walnut mortality in Colorado caused by the walnut twig beetle and thousand cankers disease. Plant Health Prog. 10.

Trevors, J. T. 1996. Sterilization and inhibition of microbial activity in soil. J. Microbiol. Methods 26:53-59.

Utley, C., Nguyen, T., Roubtsova, T., Coggeshall, M., Ford, T. M., Grauke, L. J., Graves, A. D., Leslie, C. A., McKenna, J., Woeste, K., Yaghmour, M. A., Seybold, S. J., Bostock, R. M., and Tisserat, N. 2013. Susceptibility of walnut and hickory species to Geosmithia morbida. Plant Dis. 97:601-607.

van Gils, S., Tamburini, G., Marini, L., Biere, A., Van Agtmaal, M., Tyc, O., Kos, M., Kleijn, D., and Van Der Putten, W. H. 2017. Soil pathogenaphid interactions under differences in soil organic matter and mineral fertilizer. PLoS One 12:e0179695.

Van Loon, L. C. 1997. Induced resistance in plants and the role of pathogenesis-related proteins. Eur. J. Plant Pathol. 103:753-765.

Vargas Gil, S., Pastor, S., and March, G. J. 2009. Quantitative isolation of biocontrol agents Trichoderma spp., Gliocladium spp. and actinomycetes from soil with culture media. Microbiol. Res. 164:196-205.

Venables, W. N., and Ripley, B. D. 2002. Modern Applied Statistics with S. Springer, New York, NY, U.S.A.

White, T. J., Bruns, T. D., Lee, S., and Taylor, J. 1990. Amplification and direct sequencing of fungal ribosomal RNA genes for phylogenetics. Pages 315-322 in: PCR Protocols: A Guide to Methods and Applications. M. A. Innis, D. H. Gelfand, J. J. Sninsky, and T. J. White, eds. Academic Press, New York, NY, U.S.A.

Wingfield, M. J., Brockerhoff, E. G., Wingfield, B. D., and Slippers, B. 2015. Planted forest health: The need for a global strategy. Science 349:832-836.

Yang, J.-i., Ruegger, P. M., McKenry, M. V., Becker, J. O., and Borneman, J. 2012. Correlations between root-associated microorganisms and peach replant disease symptoms in a California soil. PLoS One 7:e46420.

Yuan, J., Zhao, J., Wen, T., Zhao, M., Li, R., Goossens, P., Huang, Q., Bai, Y., Vivanco, J. M., Kowalchuk, G. A., Berendsen, R. L., and Shen, Q. 2018. Root exudates drive the soil-borne legacy of aboveground pathogen infection. Microbiome 6:156.

Zhao, J., Zhou, X., Jiang, A., Fan, J., Lan, T., Zhang, J., and Cai, Z. 2018. Distinct impacts of reductive soil disinfestation and chemical soil disinfestation on soil fungal communities and memberships. Appl. Microbiol. Biotechnol. 102:7623-7634. 